\title{
Production of Charged Higgs Bosons in a 3-3-1 Model at the CERN LHC
}

\author{
A. Alves ${ }^{1}$, E. Ramirez Barreto ${ }^{2}$, A. G. Dias ${ }^{2}$ \\ ${ }^{1}$ Departamento de Ciências Exatas e da Terra, \\ Universidade Federal de São Paulo, \\ Diadema - SP, 09972-270, Brasil \\ and \\ ${ }^{2}$ Centro de Ciências Naturais e Humanas, UFABC, \\ Santo André - SP, 09210-170, Brasil
}

(Dated: August 25, 2018)

\begin{abstract}
We perform a study of the charged Higgs production from an $S U(3)_{C} \otimes S U(3)_{L} \otimes U(1)_{X}$ model with right-handed neutrinos, postulating a custodial symmetry which reduces the number of free parameters in the scalar potential. We compute the cross sections for charged scalars for typical and new production modes. One of the new $S U(3)_{L}$ neutral gauge bosons, $Z^{\prime}$, affects some production cross sections distinguishing the model from other standard model extensions like, for example, the minimal supersymmetric standard model and general two-Higgs doublets models. The interplay between the Higgs sector of the model and that $Z^{\prime}$ gauge boson enhances substantially all the production rates of the lightest charged Higgs boson, $H_{1}^{ \pm}$, at hadron colliders compared to the MSSM. We found that a large portion of the parameters space can be probed at the LHC running at $14 \mathrm{TeV}$ center-of-mass energy in the associated $p p \rightarrow W^{ \pm} H_{1}^{\mp}+X$ production channel in the low luminosity run stage of the experiment.
\end{abstract}

PACS numbers: 


\section{INTRODUCTION}

The existence of a charged elementary scalar particle implies necessarily an extended Higgs sector beyond the standard model (SM) and, thus, an imprint for new physics. Signals of charged elementary scalars production and effects related with such particles are an important part of the research at the colliders. This is well justified because searching for experimental proof of fundamental scalars is a necessary step to establish which of the many new proposed theories will be able to explain the experimental facts.

Charged scalars are part of spectrum of several models for physics beyond the SM. But the investigations realized until now have mainly been concentrated in the two-Higgs doublet model (2HDM) and the minimal supersymmetric standard model (MSSM). At present, the most stringent experimental constraints for the mass of a charged scalar, which we denote generically as the $H^{ \pm}$, comes from LEP-II and from Tevatron direct searches. Considering 2HDM the experiments yielded the mass constraint $M_{H^{ \pm}}^{2 H D M} \geq 78.6 \mathrm{GeV}$ for the charged Higgs boson, considering decays only into the channels $H^{+} \rightarrow c \bar{s}$ and $\tau^{+} \nu$, [1]. For the MSSM, we have the result from the CDF Collaboration stating that no signal was found in the mass region $80 \mathrm{GeV} \leq M_{H^{ \pm}}^{\text {susy }} \leq 160 \mathrm{GeV}$ for the charged Higgs boson [2].

The ATLAS and the CMS Collaborations have made studies about the production and detection of charged Higgs considering the pair production, and the associated production with the quark top or with the charged gauge boson [3], [4]. These studies have shown that for the LHC energy and luminosities, the charged Higgses could have a significant potential for discovery. It gives additional motivation to perform analyses, in the LHC context, of charged scalars predicted by models different from the 2HDM and the MSSM.

Interesting extensions of the SM are the class of models based on $S U(3)_{C} \otimes S U(3)_{L} \otimes U(1)_{X}$ group, known as 3-3-1 models [5],[7],[6],[8]. These models have a symmetry breakdown pattern $S U(3)_{C} \otimes S U(3)_{L} \otimes U(1)_{X} / S U(3)_{C} \otimes S U(2)_{L} \otimes U(1)_{Y}$ which can be connected to a scalar field condensation at the $\mathrm{TeV}$ scale. The first proposals of these models were constructed considering three triplets of scalar fields, but a construction with two triplets of scalar fields is possible as well [9]. Taking into account the symmetry breakdown, 8 degrees of freedom from the scalars fields are incorporated as longitudinal components of the massive gauge bosons. Therefore, there are more than one physical scalar left in the particle spectrum of these models, with at least one charged state. The characteristics of 
the charged scalars, like electric charge and couplings, depend on the specific model version. Detailed analysis of the interactions and production are then crucial in order to validated or rule out some of these models.

Besides coinciding with the SM at low energies, these models have several attractive features. Some of them are the following: the anomalies cancellation occur only when the number of generations are a multiple of three, and assuming asymptotic freedom in QCD it is concluded that there must be three generations; the $S U(3)_{L}$ symmetry restricts the electroweak mixing angle $\theta_{W}$, furnishing a hint for an explanation of its value, since $\sin \theta_{W}<0.25$ in the models of Refs. [5, 7], and $\sin \theta_{W}<0.375$ in the models of Refs. [6, 8]; also, quantization of the electric charge can be explained in the context of these models [10].

These and other features have motivated many studies concerning the 3-3-1 models. For example, new gauge bosons are predicted by these models and production analysis of such particles has been investigated in several aspects in Refs. [11] showing, in general, a great potential for discovering at the LHC and linear colliders. For other phenomenological issues involving the 3-3-1 models see [12].

The version in focus here is known as 3-3-1 model with right-handed neutrinos (3-31RHN). It has a new neutral fermionic field, with the usual charged and neutral fields, for completing each leptonic triplet representation [8], [6]. In order to break the symmetry three scalar triplets are taken into account. In principle, those triplets may form several invariant operators through multiplication resulting in a great set of free parameters. In face of it we, additionally, impose a sort of global custodial symmetry which reduces the number of free parameters in the scalar potential [13].

Our main goal was to investigate in the 3-3-1RHN the lightest charged Higgs boson production at hadron colliders. We analyzed several production channels of phenomenological interest including Higgs pairs, associated production to $W$ bosons, associated production to top quarks, and from top quark decay. As a result we find that the cross sections for all these processes are at least as high as the MSSM analogues. The presence of the new gauge boson $Z^{\prime}$ of the model affects some of the production processes that we have studied endowing the model some distinguishing features compared to other standard model extensions. In particular, we found that the process $p p \rightarrow W^{ \pm} H_{1}^{\mp}+X$ at the $14 \mathrm{TeV}$ LHC allows, through a reconstruction analysis, a clear identification of the charged Higgs boson decaying to a top and a bottom quark. 
This paper is organized as follows: In Sec. II, we present briefly the 3-3-1RHN version and discuss the reduced potential and the scalar spectrum. The decay channels and the production of the charged Higgs at the LHC are shown in the Sec. III. In the Sec. IV, we comment our results involving the decay of the charged scalar and the gauge boson. Finally, we present our conclusions in Sec. V.

\section{DESCRIPTION OF THE MODEL}

In what follows we resume the representation content of the model and its essential aspects necessary for developing our work.

The lepton triplets are composed by two neutral fields and a charged lepton

$$
\Psi_{a L} \equiv\left[\begin{array}{lll}
\nu_{a L} & e_{a L} & N_{a L}
\end{array}\right]^{T} \sim(3,-1 / 3)
$$

with the right-handed singlets

$$
e_{a R} \sim(\mathbf{1},-1)
$$

where $a=1,2,3$ is the family index; the numbers in parentheses refer to the transformation properties under $S U(3)_{L}$ and $U(1)_{X}$ (the color quantum number will be omitted), respectively; $N_{a L}$ are new neutral lepton fields. Right-handed neutrinos could be added, but they are not relevant for the developments here. For the quarks,

$$
\begin{aligned}
& Q_{m L} \equiv\left[\begin{array}{lll}
d_{m L} & u_{m L} & D_{m L}
\end{array}\right]^{T} \sim\left(\mathbf{3}^{*}, 0\right), \\
& Q_{3 L} \equiv\left[\begin{array}{lll}
u_{3 L} & d_{3 L} T_{L}
\end{array}\right]^{T} \sim(\mathbf{3}, 1 / 3), \\
& u_{\alpha R} \sim(\mathbf{3}, \mathbf{1}, 2 / 3), \quad d_{\alpha R} \sim(\mathbf{1},-1 / 3), \\
& T_{R} \sim(\mathbf{3}, \mathbf{1}, 2 / 3), \quad D_{m R} \sim(\mathbf{1},-1 / 3),
\end{aligned}
$$

where $m=1,2$ and $\alpha=1,2,3 . D_{m}$ and $T$ are new quark fields. Anomalies cancellation requires $Q_{m L}$ to be an $S U(3)_{L}$ antitriplet. Scalar fields are such that they form the following triplets

$$
\begin{aligned}
& \eta \equiv\left[\begin{array}{lll}
\eta^{0} & \eta^{-} & \eta^{\prime 0}
\end{array}\right]^{T} \sim(\mathbf{3},-1 / 3) \\
& \rho \equiv\left[\begin{array}{lll}
\rho^{+} & \rho^{0} & \rho^{\prime+}
\end{array}\right]^{T} \sim(\mathbf{3}, 2 / 3) \\
& \chi \equiv\left[\begin{array}{lll}
\chi^{0} & \chi^{-} & \chi^{\prime 0}
\end{array}\right]^{T} \sim(\mathbf{3},-1 / 3)
\end{aligned}
$$


In order to restrict the scalar fields self-interactions it is assumed an approximate global symmetry $S U(3)_{L^{\prime}} \otimes S U(3)_{R^{\prime}}$ from which we define the tritriplet

$$
\Phi=(\eta \rho \chi)
$$

This object transforms under the global symmetry as follows

$$
\Phi \rightarrow \Omega_{L^{\prime}} \Phi \Omega_{R^{\prime}}^{\dagger}
$$

and the invariant potential, containing operators up to dimension four, is

$$
\begin{aligned}
V(\Phi) & =\mu^{2} \operatorname{Tr}\left(\Phi^{\dagger} \Phi\right)+\frac{f}{2} \epsilon_{i j k} \epsilon_{l m n}\left(\Phi_{i l} \Phi_{j m} \Phi_{k n}+H . c .\right) \\
& +\lambda_{1}\left[\operatorname{Tr}\left(\Phi^{\dagger} \Phi\right)\right]^{2}+\lambda_{2} \operatorname{Tr}\left(\Phi^{\dagger} \Phi\right)^{2}
\end{aligned}
$$

Both gauge $U(1)_{X}$ and Yukawa couplings break explicitly $S U(3)_{L^{\prime}} \otimes S U(3)_{R^{\prime}}$. This is the reason we treat it as an approximated global symmetry. It has the important consequence of allowing us to have different vacuum expectation values (VEV) for some of the neutral components in the scalar triplets giving, in this way, a consistent pattern for breakdown of gauge symmetries. Assuming the vacuum expectation values $\left\langle\eta^{0}\right\rangle=v / \sqrt{2},\left\langle\rho^{0}\right\rangle=u / \sqrt{2}$, and $\left\langle\chi^{\prime 0}\right\rangle=w / \sqrt{2}$ the constraint equations for minimum condition of the potential are as follow;

$$
\begin{aligned}
& \lambda_{1}\left(w^{2}+v_{w}^{2}\right)+\lambda_{2} v^{2}+\frac{\sqrt{2}}{4 v} f u w=-\mu^{2}-\delta \mu_{1}^{2} \\
& \lambda_{1}\left(w^{2}+v_{w}^{2}\right)+\lambda_{2} u^{2}+\frac{\sqrt{2}}{4 u} f v w=-\mu^{2}-\delta \mu_{2}^{2} \\
& \lambda_{1}\left(w^{2}+v_{w}^{2}\right)+\lambda_{2} w^{2}+\frac{\sqrt{2}}{4 w} f u v=-\mu^{2}-\delta \mu_{3}^{2}
\end{aligned}
$$

where $v_{w}^{2}=v^{2}+u^{2}\left(v_{w}=246 \mathrm{GeV}\right)$ and $\delta \mu_{i}$ are the loop corrections involving parameters of the explicitly breaking symmetry terms. Once the scalar triplets have distinct $U(1)_{X}$ charges and couplings with the fermionic fields the right side of the above Eqs. (17) are different, so that there can be a solution for different values of $v, u$, and $w$. Now, $\left\langle\chi^{\prime 0}\right\rangle$ realizes the break $S U(3)_{L} \otimes U(1)_{X} \rightarrow S U(2)_{L} \otimes U(1)_{Y}$ and we assume that $w$ is bigger than the $v$ and $u$. These two last values are the scales connected to the breakdown to the electromagnetic factor $S U(2)_{L} \otimes U(1)_{Y} \rightarrow U(1)_{e m}$ and, therefore, directly related to the particle masses we already known in the SM. 
We have that 8 from 18 degrees of freedom of scalar triplets turn into longitudinal polarization for massive gauge bosons $W^{ \pm}, Z, U^{ \pm}, V^{0}, V^{0 \dagger}$, and $Z^{\prime}$ resulting from symmetry breakdown. Thus, the physical scalar particle spectrum of the model has three CP even, one CP odd, one neutral complex, and two single charged scalars composing the remaining 10 degrees of freedom. We define the triplets neutral components obtaining VEV in terms of real and imaginary components as

$$
\varphi^{0}=\left\langle\varphi^{0}\right\rangle+\frac{1}{\sqrt{2}}\left(\xi_{\varphi}+i \zeta_{\varphi}\right)
$$

and taking into account a further assumption that $f=-4 \sqrt{2} \lambda_{1} w$ for simplifying our analyses we get the following mass expressions: for the three $\mathrm{CP}$ even eigenstates $h_{i}^{0}$

$$
\begin{aligned}
& M_{h_{1}}^{2}=2 \lambda_{2}(2 x+1) u^{2}, \\
& M_{h_{2}}^{2}=2 \lambda_{2}\left(2 x w^{2}+u^{2}\right), \\
& M_{h_{3}}^{2}=2 \lambda_{2}\left[(x+1) w^{2}+x u^{2}\right] ;
\end{aligned}
$$

for the CP odd eigenstate $A^{0}$

$$
M_{A^{0}}^{2}=2 \lambda_{2} x\left(v_{w}^{2} \frac{w^{2}}{u v}+u v\right)
$$

for the complex neutral eigenstate $H^{0}$

$$
M_{H^{0}}^{2}=\lambda_{2}\left(2 x \frac{u}{v}+1\right)\left(v^{2}+w^{2}\right)
$$

and for the two charged eigenstates $H_{1}^{ \pm}$and $H_{2}^{ \pm}$

$$
\begin{gathered}
M_{H_{1}^{ \pm}}^{2}=\lambda_{2}\left(2 x \frac{w^{2}}{u v}+1\right) v_{w}^{2} \\
M_{H_{2}^{ \pm}}^{2}=\lambda_{2}\left(2 x \frac{v}{u}+1\right)\left(u^{2}+w^{2}\right)
\end{gathered}
$$

where $x=\lambda_{1} / \lambda_{2}$.

To avoid any conflict caused by a tree level deviation of the $\rho$ parameter we also take a special relation between the VEVs according to

$$
u^{2}=\frac{1-2 \mathrm{sw}^{2}}{2 \mathrm{cw}^{2}} v_{w}^{2}
$$


where $\mathrm{sw}^{2}\left(\mathrm{cw}^{2}\right)$ stands for $\sin ^{2} \theta_{W}\left(\cos ^{2} \theta_{W}\right)$, with $\theta_{W}$ being the electroweak mixing angle. The relation above is obtained when we look for VEVs values which leads to a match with the tree level SM prediction $\rho=1$. Equation (16) is also what come out as a solution for the VEVs which cancels the mixing between the neutral massive gauge bosons $Z$ and $Z^{\prime}$. There are two of vacuum configurations realizing this: one is independent of the VEV $w$ value and given by Eq. (16), the other is for $w \rightarrow \infty$ with $u$ and $v$ taking any values satisfying $v_{w}^{2}=v^{2}+u^{2}$ (observe that this last configuration recovers the case of 2HDM where the tree level $\rho$ parameter is the same as in the SM). Also, as a consequence of Eq. (16) all known fermions in the model have the same gauge vector and axial couplings like in the SM [13]. Taking into account the value $\mathrm{sw}^{2} \approx 0.2321$ we have $v \approx 198.5$, and $u \approx 145.3$. With this we now take the LEP limit on the CP even eigenstate $h_{1}^{0}$, which is equivalent to the SM Higgs boson, as being $M_{h_{1}} \geq 114 \mathrm{GeV}$ in Eq. (9) to constrain the values of the parameters $\lambda_{1}$ and $\lambda_{2}$ according to

$$
2 \lambda_{1}+\lambda_{2} \geq 0.31
$$

Our focus here is on the production of the lightest charged scalar, and we apply this constraint on the tree level masses obtained above for the scalar particles. Fixing values for $\lambda_{2}$ and $w$ we show in Figs. 1, 2, 3 below the masses of the neutral scalars as a function of $x$ and in Fig. 4 the masses of the charged scalars also as a function of $x$. It is interesting to observe that our assumption of the approximate global symmetry and the defined values of $v$ and $u$ make $h_{2}^{0}, A^{0}$, and $H_{1}^{ \pm}$practically degenerated in mass, except for a small interval of values for $x$, where $A^{0}$ can be lighter than $h_{1}^{0}$. We observe at Fig. 4 that the $H_{1}^{ \pm}$are the lighter ones. Also Eq. (17) implies a lower bound of $M_{H_{1}} \geq 137 \mathrm{GeV}$ on the lightest charged scalars. We shall use this lower bound in our phenomenological analysis of the production and decay of the lightest charged Higgs bosons. 


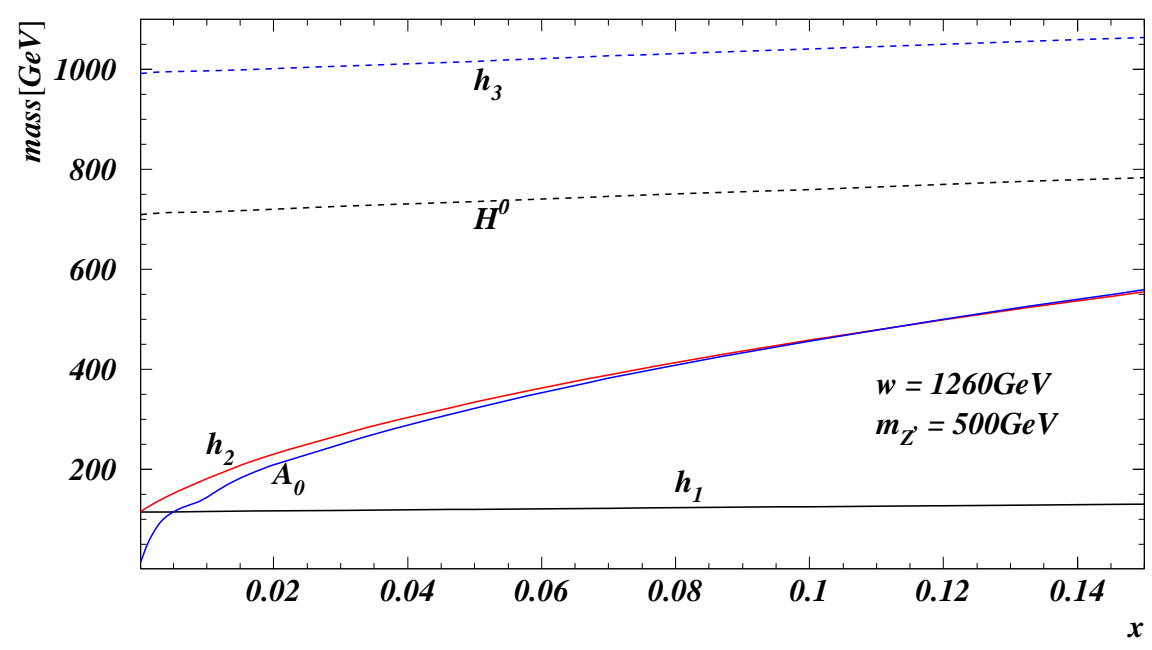

Figure 1: Masses of the neutral scalar particles $h_{i}^{0}, A^{0}$, and $H^{0}$ for $\lambda_{2}=0.31$ and $m_{Z^{\prime}}=500 \mathrm{GeV}$.

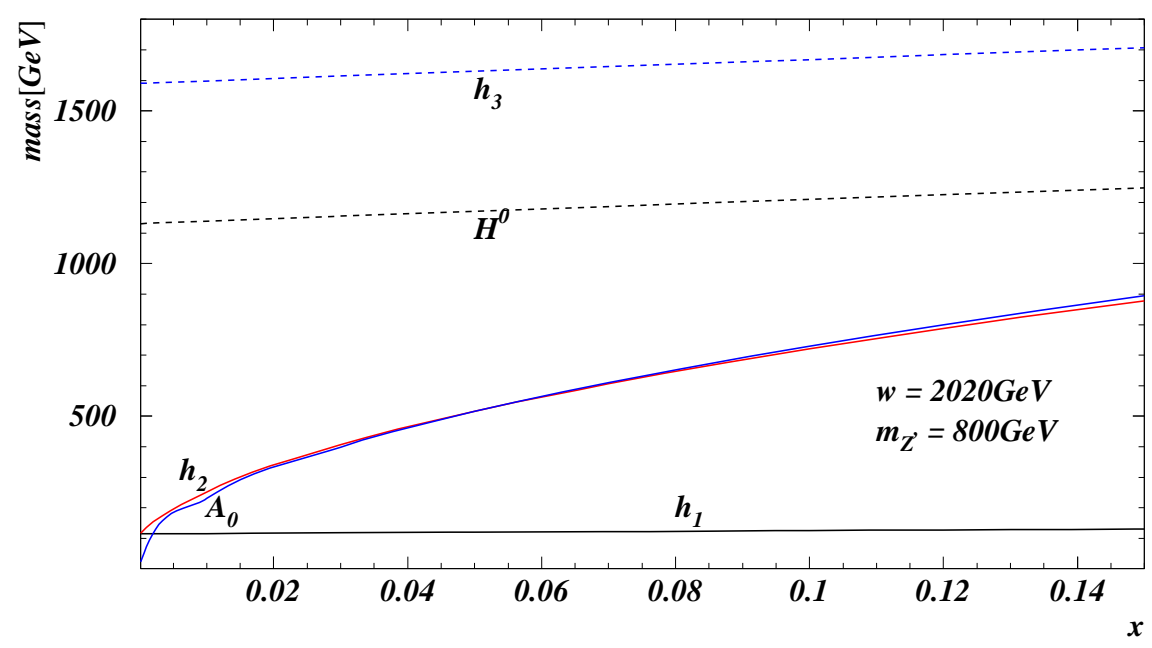

Figure 2: Masses of the neutral scalar particles $h_{i}^{0}, A^{0}$, and $H^{0}$ for $\lambda_{2}=0.31$ and $m_{Z^{\prime}}=800 \mathrm{GeV}$. 


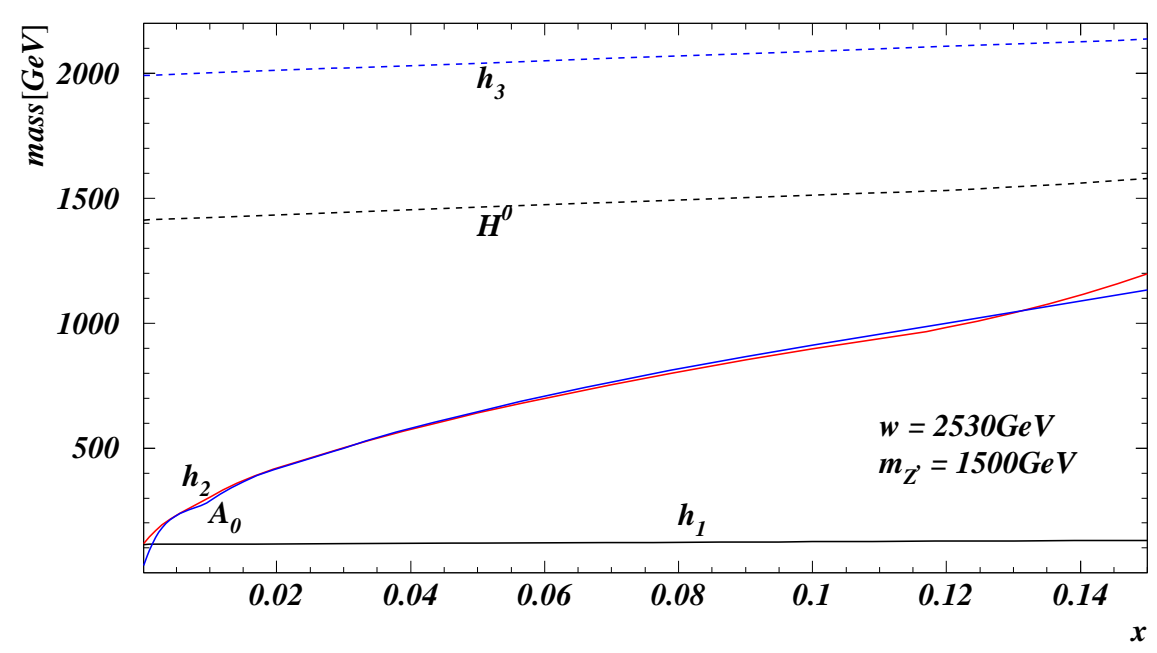

Figure 3: Masses of the neutral scalar particles $h_{i}^{0}, A^{0}$, and $H^{0}$ for $\lambda_{2}=0.31$ and $m_{Z^{\prime}}=1500 \mathrm{GeV}$.

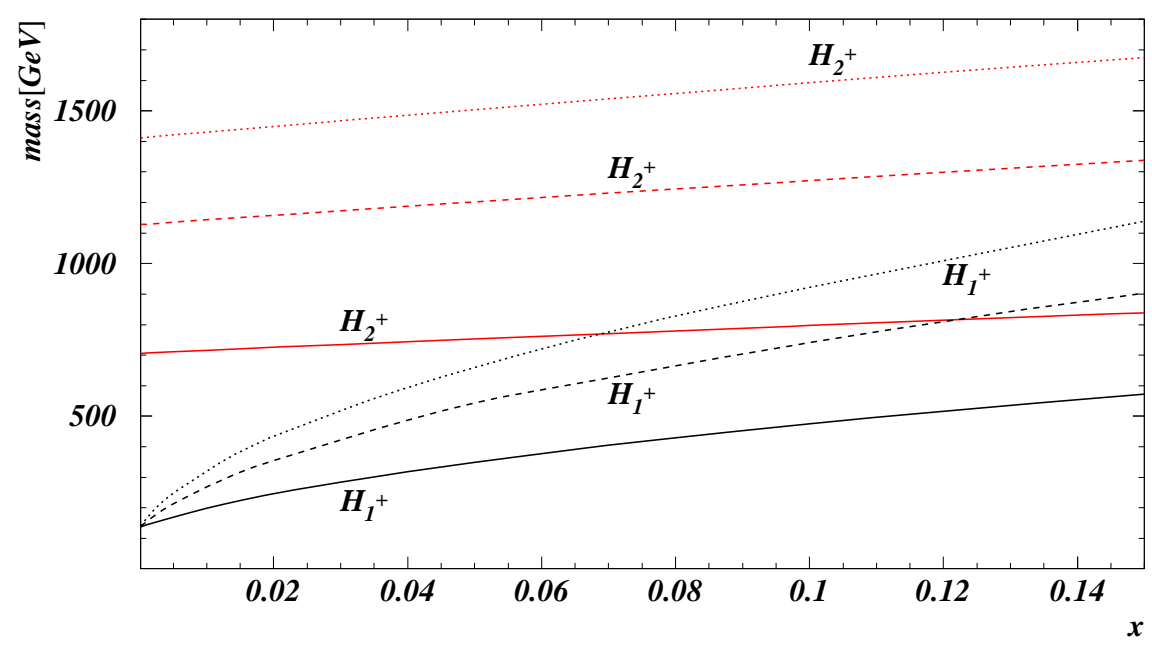

Figure 4: Masses of the charged scalar particles $H_{1}^{ \pm}$and $H_{2}^{ \pm}$for $\lambda_{2}=0.31$. The solid lines correspond to $m_{Z^{\prime}}=500$, the dashed lines to $m_{Z^{\prime}}=800 \mathrm{GeV}$, and the dotted ones to $m_{Z^{\prime}}=1500$ $\mathrm{GeV}$.

The gauge bosons $W_{\mu}^{ \pm}$and $Z$ have tree level masses as in the SM. For the new gauge 
bosons, $U^{ \pm}, V^{0}, V^{0 \dagger}$, and $Z^{\prime}$, we have the following mass expressions

$$
\begin{aligned}
& m_{U}^{2}=\frac{g^{2}}{4}\left(u^{2}+w^{2}\right) \\
& m_{V}^{2}=\frac{g^{2}}{4}\left(v^{2}+w^{2}\right) \\
& m_{Z^{\prime}}^{2}=\frac{g^{2}}{36\left(t^{2}+3\right)}\left[\left(2 t^{2}+6\right)^{2} w^{2}-\left(8 t^{4}-6 t^{2}-9\right) v_{w}^{2}\right]
\end{aligned}
$$

where we have used the definition

$$
t^{2}=\frac{g_{X}^{2}}{g^{2}}=\frac{\mathrm{sw}^{2}}{1-\frac{4}{3} \mathrm{sw}^{2}}
$$

The tree level mass expression for all new gauge bosons show dependence with $w$. This is due the fact that such new particles appear when completing the representation of $S U(3)_{L} \otimes$ $U(1)_{X}$, and this symmetry is supposedly broken at the $w$ scale.

In the Yukawa sector we assume, just for simplification, that the known quarks do not have mixing with the new quarks. This can be achieved taking into account some sort of $Z_{n}$ symmetry which preserves the scalar potential in Eq. (66). Under this assumption, the interaction Lagrangian for the known quarks with the charged Higgs $H_{1}^{ \pm}$is

$$
\begin{aligned}
-\mathcal{L}_{Y} & =\frac{g}{\sqrt{2} m_{W}} \frac{v}{u} \overline{\mathcal{U}} M_{u}\left[V_{C K M}-V_{L}^{u \dagger} \Delta V_{L}^{d}\right] P_{L} \mathcal{D} H_{1}^{+} \\
& +\frac{g}{\sqrt{2} m_{W}} \frac{u}{v} \overline{\mathcal{U}}\left[V_{C K M}-V_{L}^{u \dagger} \Delta V_{L}^{d}\right] M_{d} P_{R} \mathcal{D} H_{1}^{+}+H . c .
\end{aligned}
$$

where $\mathcal{D}=(d, s, b)^{T}, \mathcal{U}=(u, c, t)^{T}$, with $V_{C K M}=V_{L}^{u \dagger} V_{L}^{d}$ being the usual CabbiboKobayashi-Maskawa matrix defined in terms of the rotation matrices, $V_{L}^{u}$ and $V_{L}^{d}$, for the $u$ and $d$ type quarks mass eigenstates whose eigenvalues are the entries of the diagonal matrices $M_{u}=\operatorname{diag}\left(m_{u}, m_{c}, m_{t}\right), M_{d}=\operatorname{diag}\left(m_{d}, m_{s}, m_{b}\right)$ and $\Delta=\operatorname{diag}(0,0,1)$. The expressions inside brackets in Eq. (22) differ from just being $V_{C K M}$, which is obtained in 2HDMs, for example, because the 3-3-1 model has a peculiarity that the third generation of quarks transforms diferently from the other two.

It is clear from the quarks mass matrices that in order to study the interactions among the charged Higgs and the quarks, we might consider only contributions of the third generation. Then, we can write the most relevant interaction in Eq. (22) involving $H_{1}^{ \pm}$in the form

$$
-\mathcal{L}_{Y}^{t b H_{1}}=\frac{g}{\sqrt{2} m_{W}} \bar{t}\left[V_{C K M}-V_{L}^{u \dagger} \Delta V_{L}^{d}\right]_{33}\left(\frac{v}{u} m_{t} P_{L}+\frac{u}{v} m_{b} P_{R}\right) b H_{1}^{+}+H . c .
$$


The elements of the matrices $V_{L}^{u}$ and $V_{L}^{d}$ are not known and we consider here that they are

such that $\left|\left[V_{L}^{d \dagger} \Delta V_{L}^{u}\right]_{33}\right| \ll 1$. Therefore, our conclusions will be based on the fact that $H_{1}^{ \pm}$ decay mainly through a top-bottom channel, when $M_{H_{1}^{ \pm}} \geq m_{t}+m_{b}$.

It must be pointed out a similarity of the $H_{1}^{ \pm}$couplings in Eq. (23) with the corresponding ones in 2HDM. This is due the fact $H_{1}^{ \pm}$is a combination of symmetry eigenstates $\eta^{ \pm}$and $\rho^{ \pm}$in Eq. (4), with each of these last two fields belonging to two different $S U(2)_{L}$ doublets. The resemblance turns out more evident putting on Eq. (23) the usual parameter definition $\tan \beta=\frac{u}{v}=\sqrt{1-2 \mathrm{sw}^{2}}$, which in our case has a fixed value according to Eq. (16). Thus, in principle, a direct comparison with $2 \mathrm{HDM}$ could be done in these terms. But $H_{1}^{ \pm}$here has new interactions like $H_{1}^{ \pm} H_{1}^{\mp} Z^{\prime}, H_{1}^{ \pm} W^{\mp} Z^{\prime}$ which can enhance its production due $Z^{\prime}$ contribution in s-channel, for example. We shall discuss more on this in what follows.

\section{PRODUCTION OF CHARGED SCALARS $H_{1}^{ \pm}$}

The production of lightest charged scalars of the 3-3-1 model of our study can occur through the following leading modes:

1. Pair production: $q \bar{q}, b \bar{b}, g g \rightarrow H_{1}^{ \pm} H_{1}^{\mp}$

2. In association to $W$ bosons: $q \bar{q}, b \bar{b} \rightarrow H_{1}^{ \pm} W^{\mp}$

3. In association to new $Z^{\prime}$ bosons: $q \overline{q^{\prime}} \rightarrow Z^{\prime} H_{1}^{ \pm}$

4. Single production in association to top quarks: $b g \rightarrow t H_{1}^{ \pm}$

5. Single production from top quark decay: $q \bar{q}, g g \rightarrow t b H_{1}^{ \pm}$

The production modes (1),(4), and (5) are typical of extended Higgs sectors as the MSSM for instance. The associated $t H_{1}^{ \pm}$process (4) initiated by bottom quarks from the proton sea has the largest production cross section [14] in the MSSM and general 2HDMs due the $\tan \beta$ enhancement. Charged Higgs production from top quark decays (5) is important for light masses $m_{H_{1}^{ \pm}}<m_{t}+m_{b}$, and its magnitude is comparable to the top pairs production [15]. The charged Higgs pair production (1), by its turn, can be substantial for large tan $\beta$ combining all contributing channels and after including NLO corrections [16].

The mode (2) can also occur in the MSSM and 2HDM models via t-channel Yukawa diagrams involving top and bottom quarks, and through s-channel neutral Higgs bosons 
[17]. On the other hand, in the 3-3-1 models, or in models with extended gauge sectors, a new contribution is possible: through the production and subsequent decay of a new neutral gauge boson $Z^{\prime}$. In our 3-3-1 model the new interaction that makes it possible can be read from the expression Eq. (A6) in the Appendix. The same interaction is responsible for the mode (3), the $Z^{\prime} H_{1}^{ \pm}$associated production via an off shell s-channel $W$ boson. These production modes will give rise to final state topologies with at least one top quark, $b$ jets, $W$ bosons, tau leptons, and missing energy. In the next we discuss each production channel presented above in more detail.

All computations of this work were performed at tree level using the madgraph/madevent package [18] and the signals were checked against calcHep/compHep [19]. Higher order corrections were not taken into account but we comment the expected impact of QCD corrections wherever we find it is enlightening. The CTEQ6L structure functions [20] were used for the calculation of all signals and backgrounds. The factorization scale was chosen as $\mu_{F}=m_{Z^{\prime}}$ for the simulation of signal events from processes initiated by light quarks and gluons. The bottom factorization scale was chosen to be $\mu_{F} / 4$ as suggested in [21] and [16] for the sake of stability of the perturbative calculation. Different choices were made for some specific background processes and will be discussed in the appropriate moment. The renormalization scale was set equal to the factorization scale $\mu_{R}=\mu_{F}$ in all relevant processes. This choice may result in unphysical cancellations in certain cases but without a higher order analysis the precise impact of this choice is out of the scope of the study. Anyway, any cancellation would reduce the production rates which will not spoil our conclusions.

\section{A. Charged Higgs pair production}

Charged Higgs bosons can be produced in pairs through light and bottom quark annihilation and gluon-gluon fusion. The later channel receives contributions from loop diagrams with virtual heavy quarks, and the Higgses are produced via Yukawa interactions to these heavy states. In principle, all heavy quarks predicted by the theory contribute to the am-

plitude, however based on the MSSM case [16], where the gluon fusion channel contributes significantly only for rather large $\tan \beta$, we assume we can neglect that channel for the computation of total cross sections presenting conservative results.

The Drell-Yan annihilation to neutral gauge bosons and subsequent decay to Higgs pairs 
is the main production channel. The production rate can reach the hundreds of femtobarns level and the new $Z^{\prime}$ contribution is the most important one at the $14 \mathrm{TeV} \mathrm{LHC}$ as we can see in Fig. 5. As soon as the twice of the Higgs mass exceeds the $Z^{\prime}$ mass the rates drop sharply and the bottom initiated process drives the production rates.

The bottom t-channel contribution involves the enhanced Yukawa $t b H_{1}^{ \pm}$interaction which may compete with the Drell-Yan channel depending on the mass of the charged Higgs. Nevertheless, the bottom density is populated for small $\mathrm{x}$ in the proton sea, and the production of heavy states is less likely from this channel.

It is worth noting that the expected rate for the MSSM charged Higgs pair production including NLO QCD barely reaches $100 \mathrm{fb}$ for $\tan \beta=50$, as demonstrated in Ref. [16] including all possible contribution channels with little sensitivity to the specific parameter space point.

The prospects to search for charged Higgses in the pair production channel was established by the CMS and ATLAS Collaborations [3] [4], in special the charged scalars predicted by the 2HDM and the MSSM [22], [23] will be hard to detect. This conclusion can change for left-right symmetric models [24] and other 2HDM-like models as shown in [25] and where some tens of inverse femtobarns are enough for discovery at the $14 \mathrm{TeV}$ LHC. These studies can be easily adapted to a search for the charged scalars of our version of the 3-3-1 model and an extended exclusion region compared to the MSSM case is expected. We will see in the next section that the associated production of a charged Higgs and a $W$ boson is a more interesting channel to search for the $H_{1}^{ \pm}$states. 


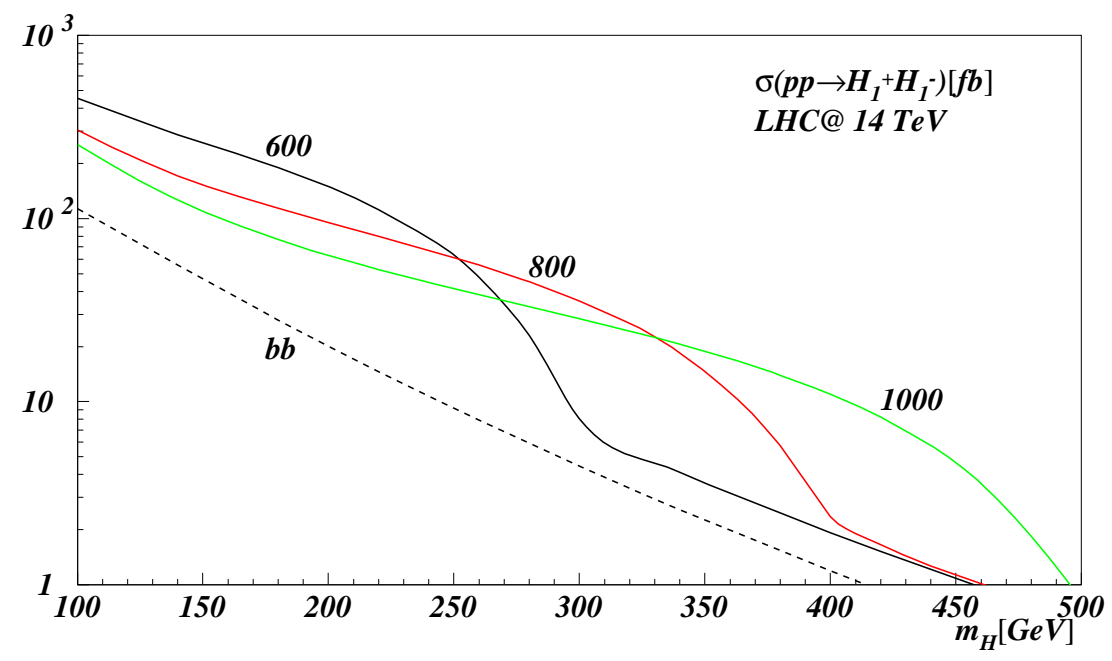

Figure 5: Production cross section (in fb) of a charged Higgs pair at the $14 \mathrm{TeV}$ LHC including the Drell-Yan contribution and the t-channel bottom initiated process (solid curves). The dashed curve shows the contribution from the Yukawa interaction only. We show the total production rates for three different $Z^{\prime}$ masses, 600, 800, and $1000 \mathrm{GeV}$.

\section{B. Associated production of a charged Higgs and a W boson}

In this section we study the associated production of a charged Higgs and a $W^{ \pm}$boson. The process occurs through the production of a $Z^{\prime}$ via $q \bar{q}$ annihilation, the $b t H_{1}^{ \pm}$Yukawa interaction via $b \bar{b}$ channel, and s-channel neutral Higgs bosons $h_{1,2}$ diagrams. As a consequence of the custodial symmetry imposed on the scalar sector of the model and the small difference between the $u$ and $v$ vacuum expectation values, the $h_{2}, A_{0}$, and $H_{1}^{ \pm}$Higgs bosons are almost degenerated in mass as we can see comparing the Figs. 1, 2, 3, and 4, As we said above, $h_{1}$ is equivalent to the SM Higgs boson and is the lightest state for practically all the parameter space. Therefore, the contribution from $b \bar{b} \rightarrow h_{1,2} \rightarrow W^{ \pm} H_{1}^{\mp}$ is negligible once the neutral Higgs bosons cannot be on their mass shell.

We show in the Fig. [6 the effective contributing channels to $W^{ \pm} H_{1}^{\mp}$ production. The charged Higgs production through an s-channel $Z^{\prime}$ is a novel feature predicted by the model that can lead to very distinctive topologies as we will see in the next section. 

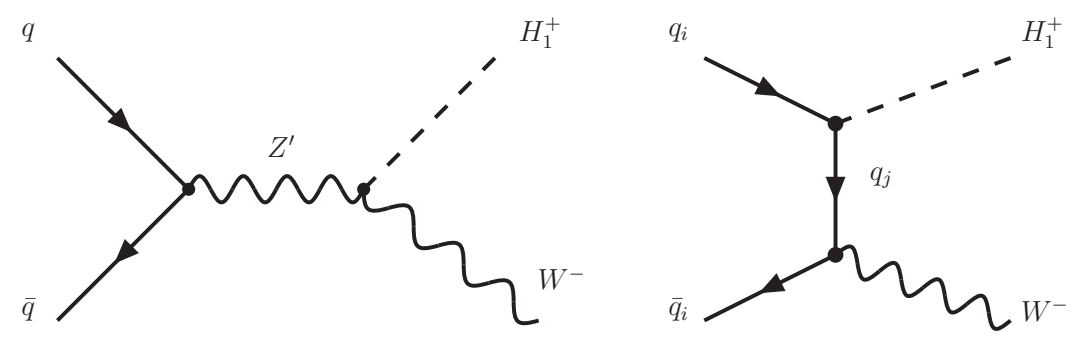

Figure 6: Feynman graphs of the contributing processes to $q \bar{q} \rightarrow W^{-} H^{+}$. At the left panel we show the $Z^{\prime}$ s-channel contribution and at the right panel the heavy quark t-channel diagram from the Yukawa interaction.

The total cross section as a function of the $Z^{\prime}$ mass, for a $300 \mathrm{GeV}$ charged Higgs, at the LHC is shown in the Fig. 7 for $\sqrt{S}=14 \mathrm{TeV}$. The bump observed at $m_{Z^{\prime}} \simeq m_{H_{1}^{ \pm}}+m_{W}$ $\mathrm{GeV}$ corresponds to the transition to on shell $Z^{\prime}$ production via the $q \bar{q}$ annihilation which is the dominant channel in that regime.

The t-channel Yukawa production is dominant when either the $Z^{\prime}$ is off shell or is very heavy as we can see at Fig. 7. Near the threshold for on shell production the contribution from the s-channel $b \bar{b} \rightarrow Z^{\prime} \rightarrow H_{1}^{ \pm} W^{\mp}$ increases to about $30 \%$ of the total $b \bar{b}$ channel, but away from the resonance this contribution is very small.

The sensitivity to the $Z^{\prime}$ mass in the t-channel Yukawa production enters through the factorization scale that was chosen as $\mu_{F}=m_{Z^{\prime}} / 4$ for the initial state bottom quarks. It has been shown [16] that choosing a smaller scale is the appropriated choice in the bottom parton picture of processes initiated by bottom quarks for the sake of the perturbative stabilization. This claim is confirmed in this case observing that the $b \bar{b}$ channel varies by a factor of 3 in magnitude in the whole range of $Z^{\prime}$ masses considered in the Fig. 8 while the factorization scale varies by a factor of 12 . The combined total cross section including positively and negatively charged states and $q \bar{q}$ and $b \bar{b}$ channels can be as high as $1.2 \mathrm{pb}$ for this charged Higgs mass.

QCD corrections are expected to be as small as the Drell-Yan processes cases and are not included in the analysis. The Yukawa t-channel contributions may have more substantial QCD corrections [16], but it is not the dominant contribution as we discussed. Nevertheless taking into account additional hard radiation may be important for a proper evaluation of signals and backgrounds in the tail of some kinematical distributions. We will return to this discussion in the next section. 
The Fig. 8 displays the $q \bar{q}$ and the $b \bar{b}$ initiated processes separately, and the total $q \bar{q}+b \bar{b}$ production rate as a function of the charged Higgs mass. The initial state bottom process is important for very light or very heavy charged Higgs masses. The contribution for a $100 \mathrm{GeV} H_{1}^{ \pm}$reaches $1 \mathrm{pb}$ and decreases by 2 orders of magnitude when $m_{H_{1}^{ \pm}}=600 \mathrm{GeV}$. This behavior is due mainly to the bottom distribution function in the proton sea. The $q \bar{q}$ contribution, by its turn, decreases sharply near the $m_{H_{1}^{ \pm}}+m_{W}$ threshold as the $Z^{\prime}$ gauge boson gets off shell, becoming smaller than the bottom initiated process.

We show in the Fig. 9 the production cross section as a function of the charged Higgs mass including all s and t-channels at the 7 and $14 \mathrm{TeV}$ LHC and at the $1.96 \mathrm{TeV}$ Tevatron for a $600 \mathrm{GeV} Z^{\prime}$ gauge boson. We can see again the threshold for $Z^{\prime}$ production near $m_{Z^{\prime}} \simeq m_{H_{1}^{ \pm}}+m_{W} \mathrm{GeV}$ and the role played by the $b \bar{b}$ channel whose contribution is important for low charged Higgs masses and in the off shell $Z^{\prime}$ regime and as the center-of-mass energy rises. We clearly see that Tevatron can only produce a charged Higgs in association with a $W$ boson through an s-channel $Z^{\prime}$. Despite the rates at the $7 \mathrm{TeV}$ LHC can reach hundreds of femtobarns we checked that the reduced integrated luminosity designed for this run precludes a statistically significant observation of a charged Higgs boson in the channel under study. We will return to this discussion later.

This large contribution of the $b \bar{b}$ channel to the total rates is a feature shared by several nonminimal Higgs sectors extensions of the SM possessing charged scalars, like the MSSM, for example. Recalling the interaction Lagrangian for $t b H_{1}^{ \pm}$vertex given by Eq. (23) we see that the factor $\frac{m_{t}}{v_{w}} \frac{v}{u}$ has a magnitude of order 1 for $m_{t}=174.3 \mathrm{GeV}$ and the vacuum expectation values as chosen in the Sec. II. A similar Lagrangian describes the charged Higgs couplings to $\tau$ leptons and $\tau$ neutrinos.

\section{Associated production of a charged Higgs and a top quark}

The initial state bottom induced process $b g \rightarrow t H_{1}^{ \pm} \rightarrow t \tau^{ \pm} \nu_{\tau}$ is, according to ATLAS [26]

and CMS [27], the most promising channel for charged Higgs bosons in two-Higgs doublets models, in particular, for the large $\tan \beta$ regime. In part this is due the large production rates expected for the process at the LHC including the $\tan \beta$ enhancement factor.

Similarly the cross section for $b g \rightarrow t H_{1}^{ \pm}$predicted by the 3-3-1-RHN model benefits the large Yukawa coupling which compensates the small $b$ quark flux from the proton sea 


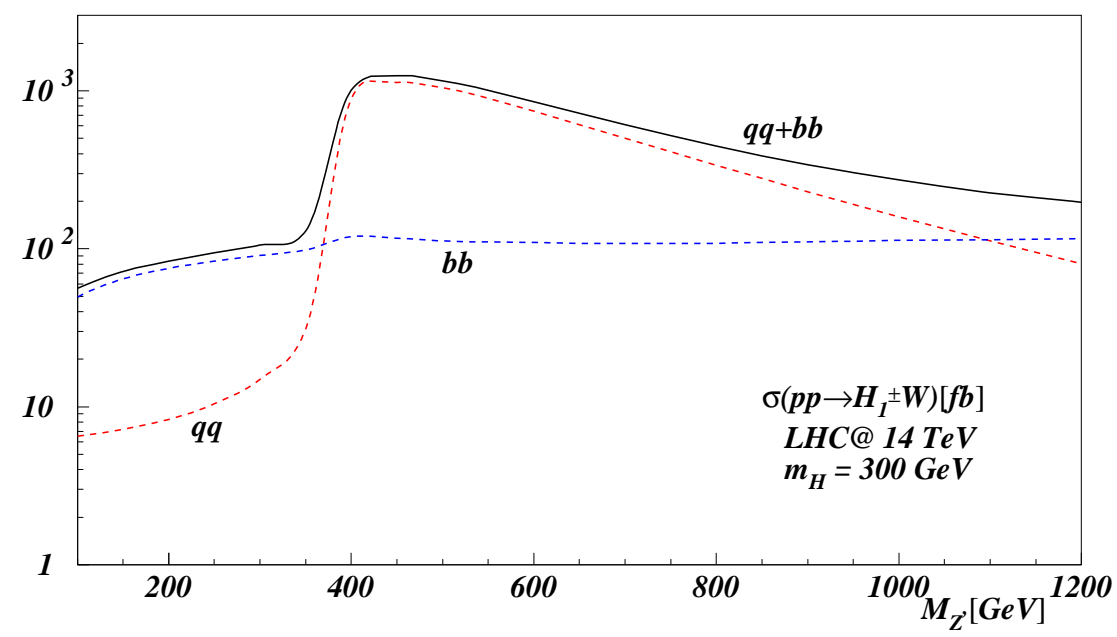

Figure 7: Total cross section (in $\mathrm{fb}$ ) for the associated $H_{1}^{ \pm}$and $W^{\mp}$ production at the LHC for $\sqrt{s}=14 \mathrm{TeV}$ as a function of the $Z^{\prime}$ mass keeping the $H_{1}^{ \pm}$mass fixed at $300 \mathrm{GeV}$. The dashed lines represent the Yukawa induced process and the light quark annihilation channel. The solid line is the sum of the two contributing channels.

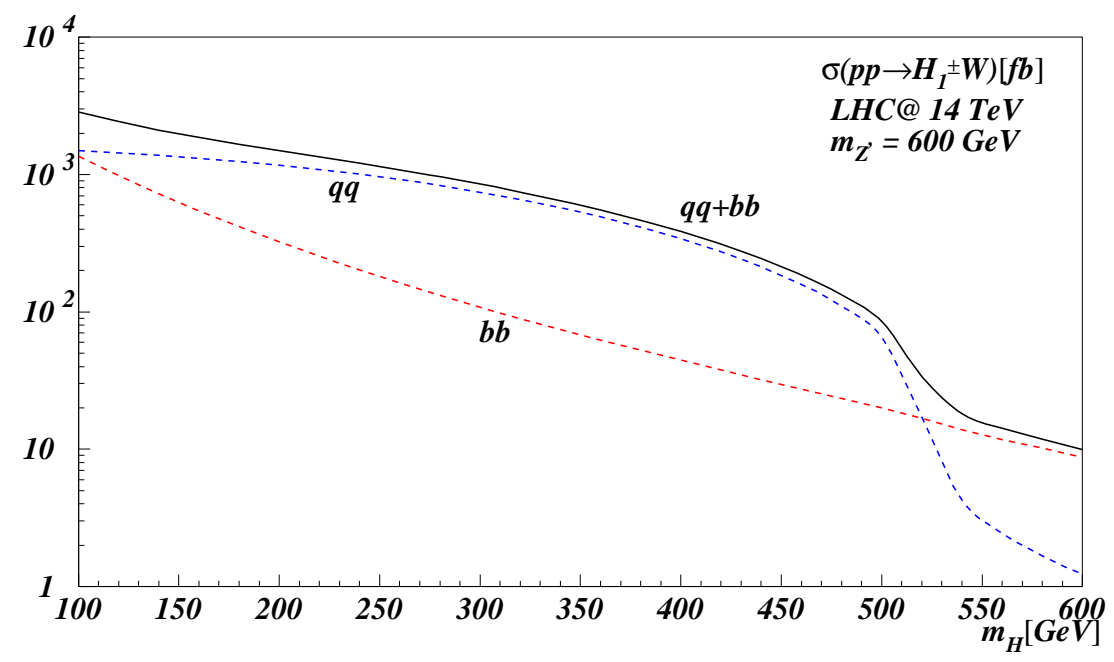

Figure 8: Total cross section (in $\mathrm{fb}$ ) for the associated $H_{1}^{ \pm}$and $W^{\mp}$ production at the LHC for $\sqrt{s}=14 \mathrm{TeV}$ as a function of the charged Higgs mass keeping the $Z^{\prime}$ mass fixed at $600 \mathrm{GeV}$. The dashed lower line represents the Yukawa induced process, and the dashed upper line the light quark annihilation channel. The solid line is the sum of the two contributing channels. 


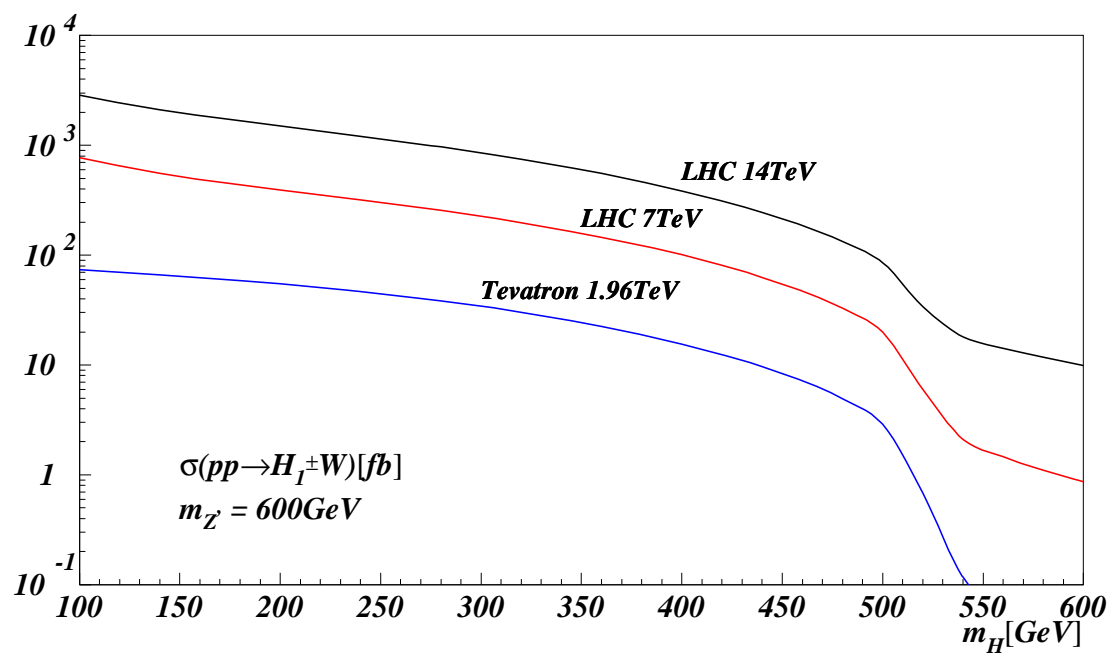

Figure 9: Total cross sections (in fb) for $W^{ \pm} H_{1}^{\mp}$ production including all the contributing channels. The upper curve shows the LHC running at $14 \mathrm{TeV}$, the middle curve the $7 \mathrm{TeV} \mathrm{LHC}$, and the lower curve the Fermilab Tevatron. The $Z^{\prime}$ mass is held fixed at $600 \mathrm{GeV}$.

resulting in large rates as we see in Fig. 10. At the LHC running at $14 \mathrm{TeV}$ the total cross section is above the picobarn level for the entire mass range considered here. This is much larger than the expected for the MSSM case with $\tan \beta=30$ and including NLO QCD corrections as shown in [14] except for small Higgs masses or very large $\tan \beta$.

At the LHC running at $7 \mathrm{TeV}$ the cross section is in the hundreds of femtobarns region for $m_{H_{1}^{ \pm}} \lesssim 400 \mathrm{GeV}$ while the rates at the Tevatron are too small as can be seen in the Fig. 10 even for small masses.

\section{Charged scalars produced in top quark decays}

Another interesting production channel is the top quark pair production with one top decaying into a bottom quark and a charged Higgs. Of course, the channel is promising only in the small Higgs mass portion of the parameter space. We show in the Fig. 11 the branching ratio of top quarks including the $b H_{1}^{ \pm}$channel. The bottom-Higgs decay can reach a $50 \%$ branching fraction for a $50 \mathrm{GeV}$ Higgs but decreases very rapidly as the mass increases. However, the branching fraction is larger than $10 \%$ if $m_{H} \lesssim 140 \mathrm{GeV}$.

The production cross section for the process $p p \rightarrow t \bar{t} \rightarrow t \bar{b} H_{1}^{+}$can be easily computed using the branching fraction information. For example, multiplying the $580 \mathrm{pb} t \bar{t}$ cross 


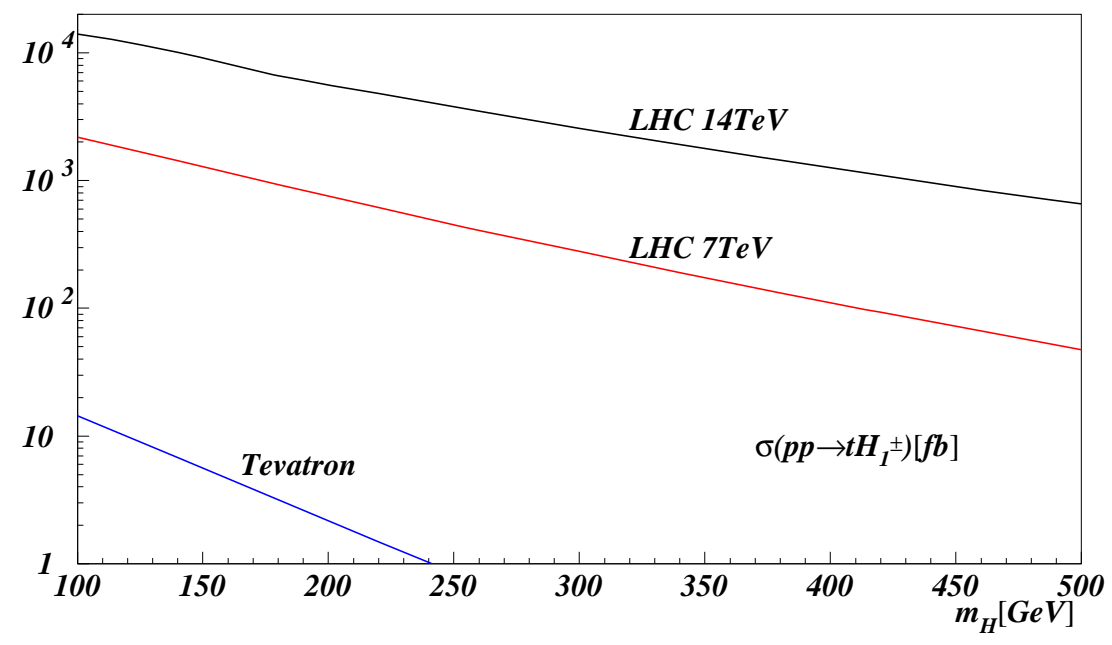

Figure 10: Associated production cross section (in $\mathrm{fb}$ ) of a top quark and a charged Higgs boson at hadron colliders as a functions of the scalar mass. The production of both positive and negative charge states were taken into account.

section at $\mathrm{LO}$ in the $\mathrm{LHC} 14 \mathrm{TeV}$ by the branching fraction of $33 \%$ for a $100 \mathrm{GeV}$ charged Higgs we find a very large rate of $191 \mathrm{pb}$. This is not a realistic estimate though because one could choose to tag the bottom quark to suppress backgrounds and in this case it is necessary to impose acceptance cuts on the bottom jet. For example, imposing the following acceptance cuts on the bottom jet

$$
p_{T}>20 \mathrm{GeV}, \quad\left|\eta_{b}\right|<2.5
$$

we have a 160 pb exclusive cross section.

Once again, the 3-3-1-RHN model predicts a larger cross section compared to the MSSM case where, for instance, the branching ratio of a $140 \mathrm{GeV}$ charged Higgs is below the 10\% level for $1 \lesssim \tan \beta \lesssim 30$ and reaches $30 \%$ for $\tan \beta \simeq 60$ [27]. For smaller masses the region around $\tan \beta=7$ still has very small branching ratios, a well know characteristics of the 2HDM, in special of the MSSM.

We have just found another distinctive feature of the Higgs sector of this 3-3-1-RHN model compared to SUSY and doublets models in general: a possibly sizable number of bottom jets plus tau leptons events associated to charged Higgs bosons production. 


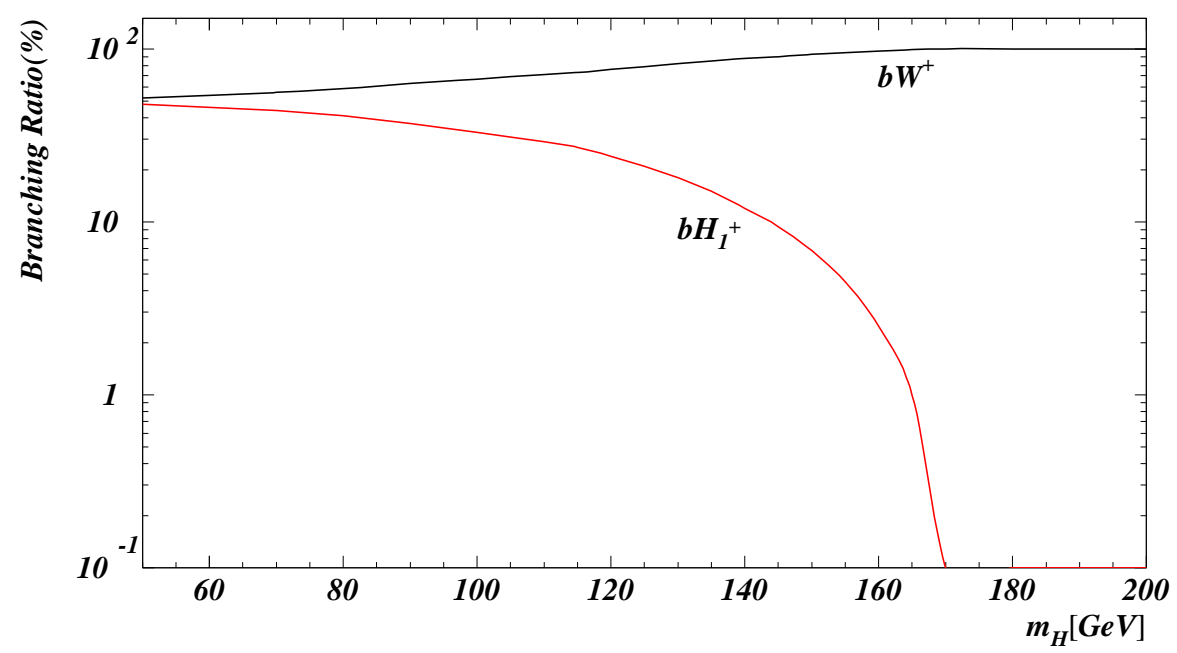

Figure 11: The branching ratios of the top quark decays into the $b W^{ \pm}$and $b H_{1}^{ \pm}$channels as a function of the charged scalar mass.

\section{E. Associated production with new neutral gauge bosons}

The production mode $p p \rightarrow Z^{\prime} H_{1}^{ \pm}$has a negligible cross section even at the $14 \mathrm{TeV}$ LHC. It is interesting to note that a $Z^{\prime}$ decaying to jets and a $H_{1}^{ \pm}$decaying taus or to $W^{ \pm} h_{1}$ may lead to a bump in the dijet mass as reported by the CDF Collaboration [28] although with a much lower cross section. Assuming a $150 \mathrm{GeV} Z^{\prime}$ and a $100 \mathrm{GeV}$ charged Higgs we found a tiny cross section of $8 \mathrm{fb}$ only. However there are versions of our model with a leptophobic $Z^{\prime}$ where this cross section can be much greater [29].

\section{PHENOMENOLOGICAL ANALYSIS FOR THE $H_{1}^{ \pm} W^{\mp}$ CHANNEL}

We demonstrate in this section the potential of the LHC, operating at $14 \mathrm{TeV}$ centerof-mass energy, to discover the lightest charged Higgs boson predicted by the model under consideration in the associated $H_{1}^{ \pm} W^{\mp}$ production mode.

Thousands of events are expected based on the production cross sections computed in the previous section in the low luminosity stages of the $14 \mathrm{TeV}$ LHC accumulating 1 to $10 \mathrm{fb}^{-1}$ of data. On the other hand 1000 events at maximum are expected at the $7 \mathrm{TeV}$ LHC after a $5 \mathrm{fb}^{-1}$ run and at the Tevatron after the whole $10 \mathrm{fb}^{-1}$ run. As we will discuss these numbers of events are not enough to claim a statistically significant discovery after 


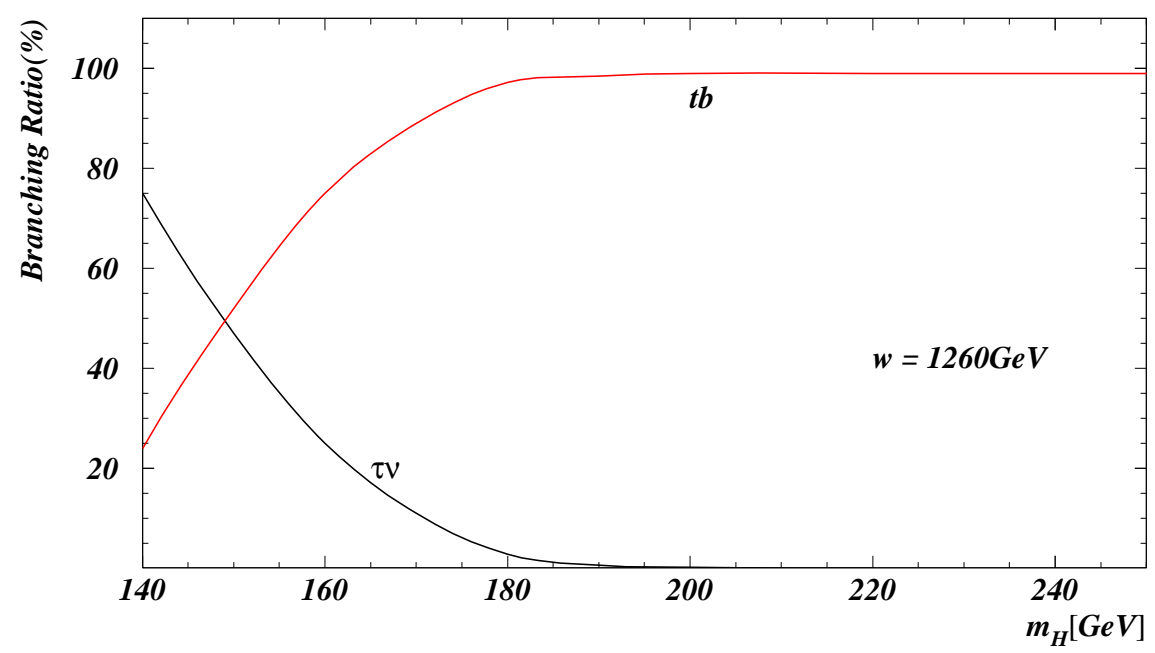

Figure 12: The branching ratios of the charged Higgs boson as a function of its mass.

background suppression based entirely on a cut method. However, an analysis based on the likelihood ratio statistics may well change this conclusion for the amount of data to be accumulated at those experiments relying on the most distinctive kinematical distributions.

The charged Higgs boson decays predominantly to top-bottom pairs for $m_{H_{1}^{ \pm}}>m_{t}+m_{b}$ and to tau leptons, $\tau^{ \pm} \nu_{\tau}$, for smaller masses as we show in the Fig. 12. This is a direct consequence of the Yukawa enhancement factor discussed in the previous section. The $Z^{\prime}$ gauge boson in its turn decays most part of the time into light quarks, about 40\% to 50\%, followed by neutrinos, bottom quarks, and charged leptons (including $\tau$ leptons). Among the heavy states, the $t \bar{t}$ channel is the most favored one with a branching ratio close to $15 \%$. The associated $H_{1}^{ \pm} W^{\mp}$ channel has a small branching ratio around $2 \%$ for Higgs masses from 500 to $1200 \mathrm{GeV}$ as can be seen in the Fig. 13 while the charged Higgs pair decay channel $Z^{\prime} \rightarrow H_{1}^{ \pm} H_{1}^{\mp}$ is very rare. Decays into heavy new fermions, gauge bosons, and neutral Higgses are negligible for the range of parameters considered in this work.

Due the large branching ratio into a bottom and a charged Higgs and the large $t \bar{t}$ production cross section at hadron colliders, the process

$$
p p \rightarrow t \bar{t} \rightarrow b \bar{b} H_{1}^{ \pm} H_{1}^{\mp} \rightarrow b \bar{b} \tau^{+} \tau^{-} \nu_{\tau} \bar{\nu}_{\tau}
$$

is expected to yield thousands of events at the LHC and the Tevatron for Higgs masses below the top mass. However, it is not possible to reconstruct the Higgses because of the 


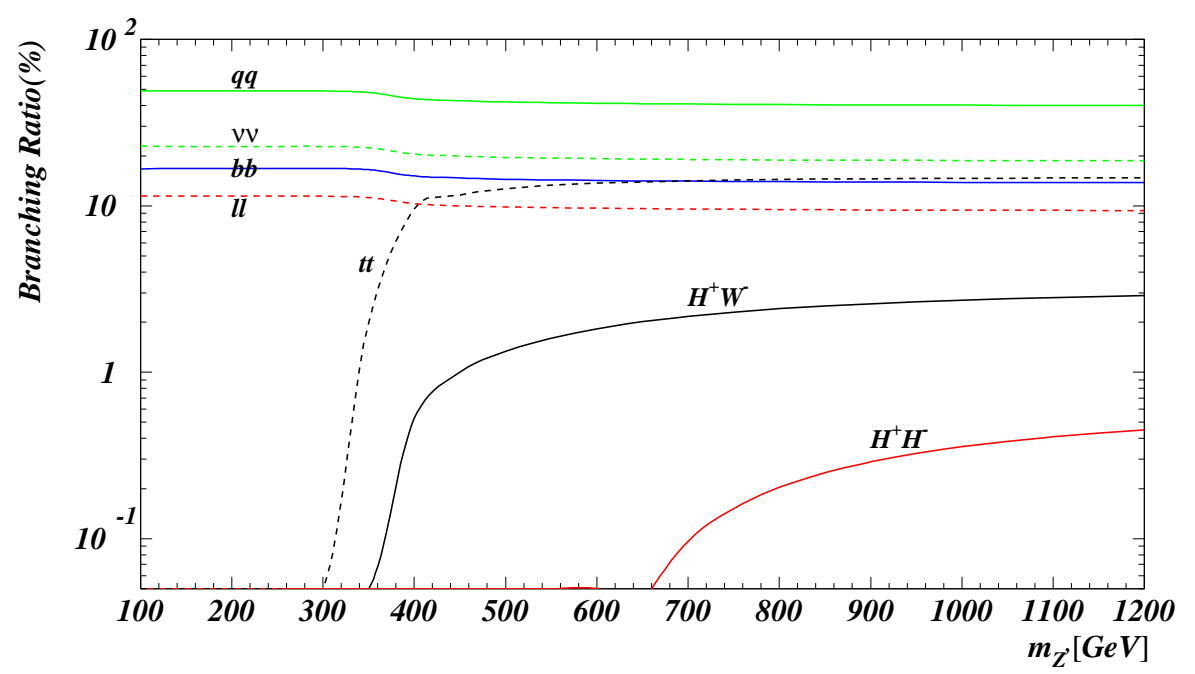

Figure 13: The branching ratios of the new neutral gauge boson $Z^{\prime}$ as a function of its mass.

escaping neutrinos. Moreover, the tau lepton decay will dilute considerably the production rates. Tagging the bottoms and taus could be a way out but the efficiency for multiple tau lepton and bottom tagging is rather small. Another possibility would be to identify a top decaying into $b W$ state and then $W \rightarrow$ jets. In this case the reconstruction is possible at the cost of a increased QCD backgrounds. Anyway, this is a classical analysis already performed in various previous works on the 2HDM and MSSM case and the known results can be applied directly to our work by an appropriate rescaling. We postpone this study to a future work.

The associated production of a top quark and the charged Higgs has the second largest cross section at the LHC as we have computed in the previous sections. Taking into account the preferred decay mode of a heavy charged Higgs, the final state configuration would be

$$
p p \rightarrow t H_{1}^{ \pm} \rightarrow t t b \rightarrow b b b+W^{+} W^{-}
$$

which could lead to a many jets plus lepton configuration or a cleaner dilepton configuration. The cleaner configuration involves two missing neutrinos which precludes the reconstruction of the charged Higgs resonance. The many jets configuration is expected to have a higher level of QCD fakes, but the reconstruction is possible. A study of this channel is currently underway [29].

Despite the smaller cross section, the associated production of a $W$ boson and the charged 
Higgs present a very distinctive signal

$$
p p \rightarrow W^{ \pm} H_{1}^{\mp} \rightarrow W^{ \pm} t b \rightarrow b \bar{b}+W^{+} W^{-}
$$

where the $W$ bosons may decay into leptons or jets. The dilepton configuration is the cleaner way to search for the charged Higgs, but again we loose the Higgs resonance. The mixed jets plus lepton state, on the other hand, permits the reconstruction up to a twofold ambiguity in the neutrino momenta. Moreover, the chain of resonances endows the signal very singular features that allow us to separate it from the SM backgrounds. The new ingredient here is the presence of the $S U(3)_{L}$ neutral gauge boson in the s-channel that decays to a $W^{ \pm} H_{1}^{\mp}$ pair. The presence of this $Z^{\prime}$ not only increases the production rates but makes the leptons and jets harder than the expected from processes induced solely by t-channel Yukawa interactions as in the usual two-Higgs doublets models.

The signal to be studied in this work is the following

$$
p p \rightarrow W^{ \pm} H_{1}^{\mp} \rightarrow b \bar{b} W^{+} W^{-} \rightarrow b \bar{b}+j j+\ell \nu_{\ell}
$$

where the light jets and leptons come from a $W$ boson decay and $\ell$ denotes an electron or muon. In order to avoid the huge QCD backgrounds we propose a double $b$-tagging assuming a $60 \%$ b-tag efficiency and a $5 \times 10^{-3}$ rejection factor against mistagged light quark and gluon jets [30]. We assume a 90\% efficiency for lepton identification and include a Gaussian smearing of the energy of jets and the lepton but not for their momentum direction.

It is important to describe more carefully the chain of resonances in order to understand our search strategy. After production, the charged Higgs boson decays to a top and bottom pair

$$
W^{ \pm}\left[H_{1}^{\mp} \rightarrow t b\right]_{A}
$$

the top quark in its turn will decay into another $W$ boson and $b$ quark, and we will have

$$
W^{ \pm}\left[H_{1}^{\mp} \rightarrow\left(t \rightarrow W^{\mp} b\right)_{B} b\right]_{A}
$$

The $W$ bosons will decay at last producing the visible particles to the detector and a neutrino, so we get at the end of the whole decay chain 


$$
\left(W^{ \pm} \rightarrow \ell^{ \pm} \nu_{\ell}\right)_{D}\left[H_{1}^{\mp} \rightarrow\left(t \rightarrow\left(W^{ \pm} \rightarrow j j\right)_{C} b\right)_{B} b\right]_{A}
$$

There are four resonant states that we labeled as $A, B, C$, and $D$. There is, of course, the primary $Z^{\prime}$ resonance; however we will not try to reconstruct the new gauge boson in our phenomenological analyses just because our main task is to unravel the presence of the charged Higgs boson. A separate work dedicated to the goal of identifying the new gauge bosons predicted by the 3-3-1RHN model is something important by itself and will not be addressed here.

The second step to explain the search strategy is listing the Standard Model backgrounds involving QCD and electroweak (EW) interactions that could mimic our signal:

1. $\mathrm{QCD}+\mathrm{EW}+Z^{\prime} t \bar{t}$ production is the most important irreducible background

2. $\mathrm{QCD}+\mathrm{EW} b \bar{b}+j j+W$ including $\mathrm{SM} Z$ bosons, photons and $\mathrm{SM} W$ bosons decaying to bottoms and jets

3. Single top quark production $t b W$

4. QCD $+\mathrm{EW} j j j j+W$ with mistagged light quark/gluon jets.

Except for the top quark pair production, where the factorization/renormalization scale was chosen to be $\mu_{F}=\mu_{R}=m_{t}$, all the other backgrounds were computed with an event-by-event factorization/renormalization scale defined by the square root of the combined transverse momentum of the identified jets, $\mu_{F}=\mu_{R}=\sqrt{\sum_{\text {jets }} p_{T}^{2}}$.

For all backgrounds and the signal we imposed the following acceptance cuts :

$$
\begin{gathered}
p_{T j, b}>30 \mathrm{GeV}, \quad p_{T \ell}>100 \mathrm{GeV} \\
\left|\eta_{j, b, \ell}\right|<2.5, \Delta R_{i k}>0.4, i, k=j, b, \ell
\end{gathered}
$$

the high $p_{T}$ cut on the leptons is a good discriminant between signal and backgrounds and an excellent experimental trigger too. On the other hand it favors the signal events resulting from the s-channel $q \bar{q} \rightarrow Z^{\prime}$ boson over the t-channel Yukawa contributions initiated by 
bottom quarks as can be seen in the Fig. 14. For this plot $m_{Z^{\prime}}=800 \mathrm{GeV}$ and $m_{H^{ \pm}}=300$ $\mathrm{GeV}$.

After acceptance cuts the signal cross section is still deeply buried beneath the backgrounds as can be read from the Table \ that shows the effect of cuts on the $m_{Z^{\prime}}=800$ $\mathrm{GeV}$ and $m_{H^{ \pm}}=300 \mathrm{GeV}$ signal and backgrounds. To further suppress the backgrounds we impose a second set of cuts exploring the fact that our signal events can be much harder than the backgrounds depending on the $Z^{\prime}$ mass

$$
H_{T}>500 \mathrm{GeV}, \quad E_{\text {Tmiss }}>60 \mathrm{GeV}
$$

where $H_{T}=\sum p_{T}$ of all hadrons. In fact the $Z^{\prime}$ events are also much harder than the t-channel Yukawa contributions as we observe in the Fig. 14, At this point the t-channel bottom initiated process contributes only $3 \%$ of the total rates. Based on this analysis we can understand why the MSSM analogue process is not a good search channel for charged Higgses. In the MSSM and 2HDM in general, the $W^{ \pm} H_{1}^{\mp}$ final state can be produced via t-channel Yukawa interactions to heavy quarks and s-channel neutral Higgses contributions both initiated by bottom quarks from gluon splittings. As a result only charged Higgses decaying to tau leptons in the large $\tan \beta$ regime can be detected at a $5 \sigma$ significance level [31].

The $H_{T}$ distribution for signal and backgrounds from Fig. 15 confirms our expectation that heavier $Z^{\prime}$ bosons are much easier to separate from backgrounds. In the subsequent analyses we do not try to optimize the cuts to take advantage of these features instead we would rather keep the analyses as independent of the particular parameter space point as possible. It is important to point out though that several improvements can be embodied in a more complete analysis.

Even after imposing such hard set of cuts on the candidate events, the backgrounds are big enough to preclude any significant conclusion as can be read from the second row of Table I.

The combined $\mathrm{QCD}+\mathrm{EW}+Z^{\prime} t \bar{t}$ production rate at the $14 \mathrm{TeV} \mathrm{LHC}$ is around $580 \mathrm{pb}$, almost 3 orders of magnitude larger than our signal. The need to suppress those backgrounds led us to choose searching for the resonance $A$, associated to the charged Higgs decay, into a purely hadronic channel. We did not take into account the semileptonic top quarks for 

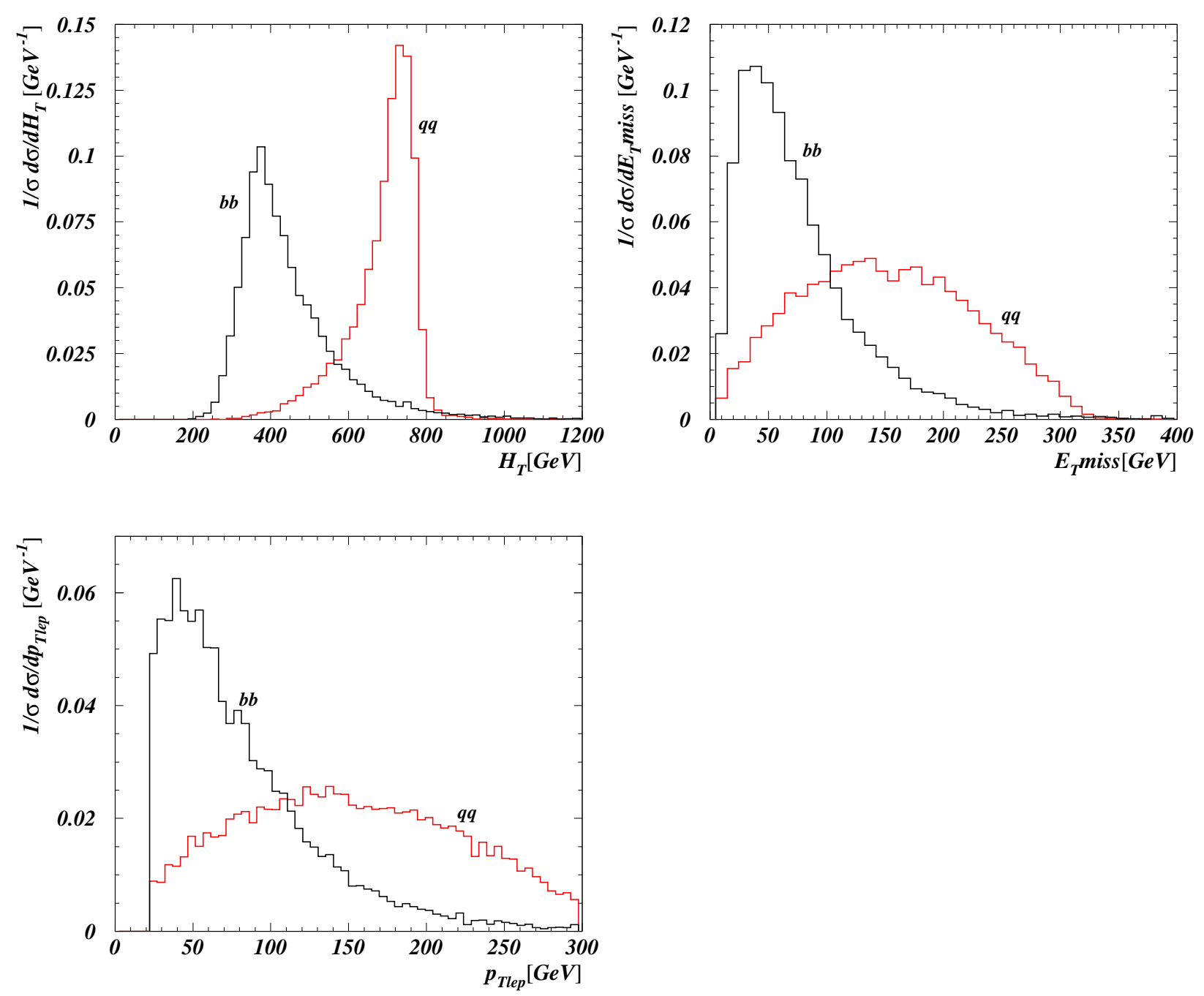

Figure 14: The left upper panel, the right upper panel, and the lower panel display the $H_{T}$ of the defined jets, the missing $E_{T}$ due the escaping neutrinos, and the transverse momentum $p_{T}$ of the charge lepton, respectively, for the signal particles produced in the $q \bar{q} \rightarrow Z^{\prime} \rightarrow W^{ \pm} H_{1}^{\mp}$ and $b \bar{b} \rightarrow W^{ \pm} H_{1}^{\mp}$ with a t-channel top. The distributions are normalized by the total cross section. The mass of the $Z^{\prime}$ and the charged Higgs are $800 \mathrm{GeV}$ and $300 \mathrm{GeV}$, respectively.

our signal. Despite we loose half of our total number of signal events, this give us a tool to explore an important advantage: the signal contains only one final state top quark.

The possibility to reconstruct the hadronic and the semileptonic tops from the $t \bar{t}$ background gives us the opportunity to tag the semileptonic tops and reject them. This can be easily done demanding a cut on the bottom-lepton invariant mass. First of all we look for the bottom quark from the hadronic top and jets from a $W$ boson, let us call it the first 


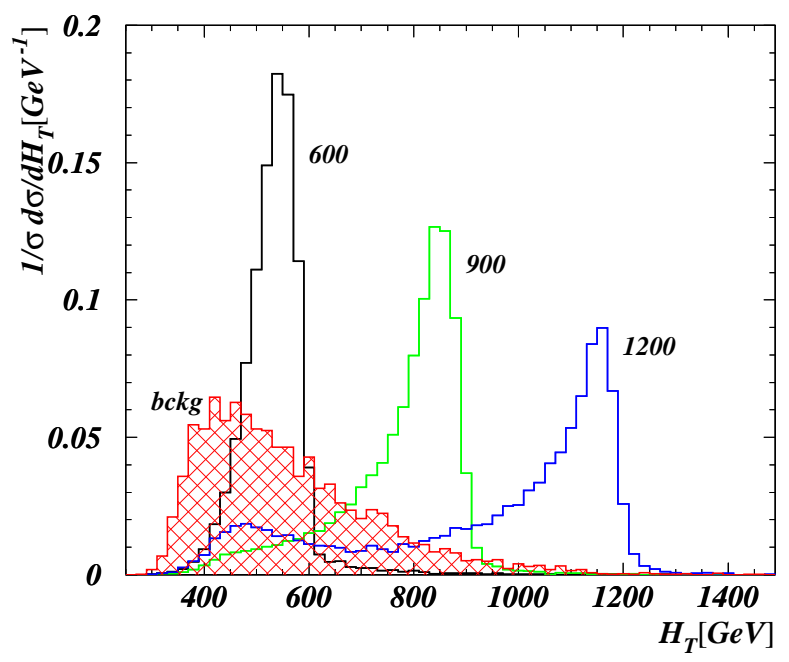

Figure 15: The normalized $H_{T}$ distributions for the defined jets from signal and background events. The signal distributions are shown for a $Z^{\prime}$ gauge boson mass of 600, 800, and $1200 \mathrm{GeV}$, respectively.

bottom quark, then we impose the following invariant mass constraints

$$
\left|m_{j j}-m_{W}\right|<20 \mathrm{GeV}, \quad\left|m_{j j b}-m_{t}\right|<20 \mathrm{GeV}
$$

The first one selects jets from $W$ boson decays and helps to clean the QCD backgrounds with at least two final state jets. The second one rejects all processes not related to top quark decays. Until this point we have made use of two resonance structures from Eq. 31, namely, $B$ and $C$.

The second signal bottom quark that comes from the charged Higgs decay is not correlated to the lepton from $W$, whereas the second $b$ quark from the $t \bar{t}$ background is the yield of the top quark that decays semileptonicaly. Thus we expect that the $t \bar{t}$ background events show and end point structure in the invariant mass of second bottom quark and the charged lepton $m_{b l}<\sqrt{m_{t}^{2}-m_{W}^{2}} \cong 160 \mathrm{GeV}$ as can be seen in the Fig. 16.

Imposing the cut

$$
m_{b l}>180 \mathrm{GeV}
$$

eliminates almost all the $t \bar{t}$ events while diluting the signal by a factor of 0.69 only for our benchmark example. We clearly see that heavier $Z^{\prime}$ bosons and lighter charged Higgses 


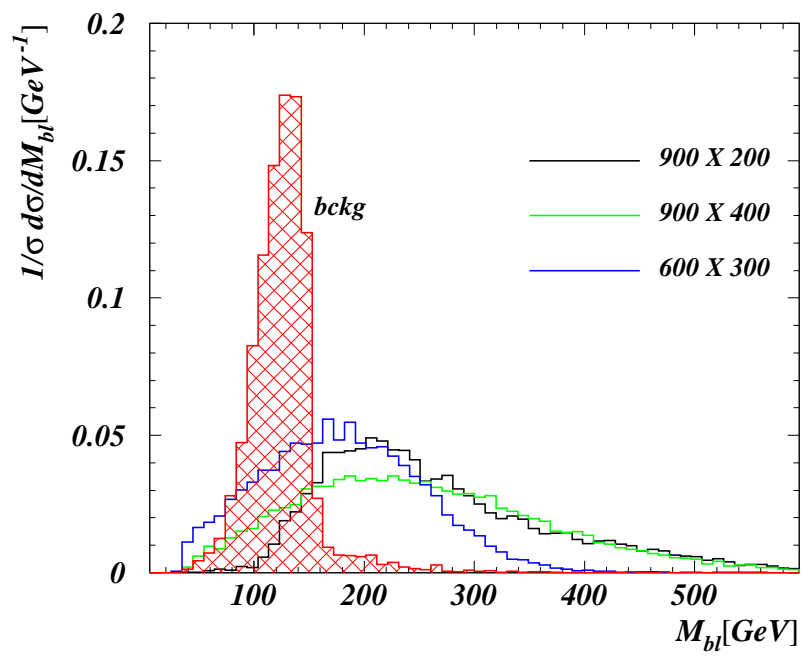

Figure 16: The second bottom (as defined in the text) and the charged lepton invariant mass distribution of the signal and the combined backgrounds. At this point the backgrounds are still dominated by the $t \bar{t}$ contribution. The distributions are normalized by the total cross section. The mass $Z^{\prime}$ and the charged Higgs are shown in the figure.

present the harder spectrum and are favored by our analysis. We show in the fourth row of the Table \ the impact on the dominant $t \bar{t}$ background and the signal. We can also observe the large impact of this cut on the $\mathrm{EW} W W Z+W Z Z$ backgrounds.

The requirement of on shell production of $W$ bosons and hadronic top quarks plus the tagging of the semileptonic tops is very effective against SM backgrounds from top pairs and electroweak gauge boson production. However there is another source of background events which is not so severely affected by those cuts. The single top process has a topology similar to our signal: one single top decaying to hadrons, and a second bottom and a charged lepton not correlated to a top quark decay. This similarity turns the single top background the dominant one after the semileptonic top veto. The $79.1 \mathrm{fb}$ background that we read from the second column of Table $\llbracket$ is the due almost entirely the contribution of the single top process.

If the $Z^{\prime}$ boson is much heavier than the charged Higgs we should expect that a high boost will collimate the hadronic top and the second bottom quark (originated from the Higgs decay) into a fat jet. That is what we precisely observe in the Fig. 17 which displays the distance distribution between the hadronic tagged top and the second bottom, $\Delta R_{t b}$. 


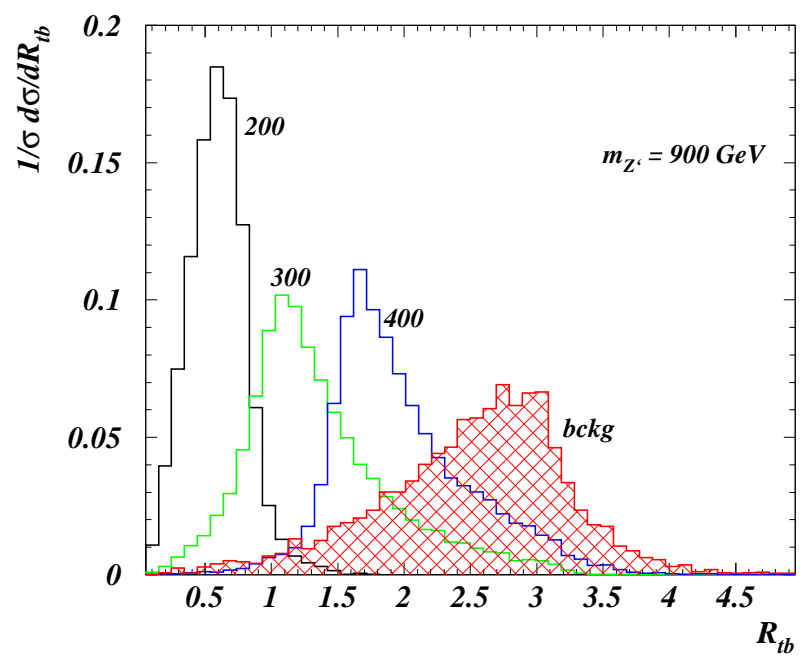

Figure 17: The normalized distribution on the distance between the hadronically tagged top quark and the second bottom jet for three different Higgs masses and a $Z^{\prime}$ mass of $800 \mathrm{GeV}$, and the combined backgrounds. The single top channel is the dominant contribution after the semileptonic top veto.

As the difference $m_{Z^{\prime}}-m_{H_{1}^{ \pm}}$increases the Higgs jet gets narrower, whereas the top-bottom pairs from the single top background are much more separated. To suppress single tops we impose an additional cut on the $\Delta R_{t b}$ variable

$$
\Delta R_{t b}<1.6
$$

This cut efficiently dilutes the remaining backgrounds by a factor of 0.15 , whereas its impact on the signal is small for our benchmark point. It should be pointed out though that this cut may impact much more strongly the signal if the difference between the $Z^{\prime}$ and the charged Higgs masses decreases. For heavy masses this negative impact can be compensated by hardening the transverse momentum cuts.

We also checked that there exists many good discriminant distributions that could be used to disentangle signal from backgrounds in specific points of the parameters space. We did not try to optimize our analysis for specific regions; instead we show that requiring only trigger cuts planned to capture possible new physics signals and exploring tagging techniques for SM and new particles is just enough to observe the charged Higgses of the model.

The $W+4 j$ where all jets originate from QCD radiation is huge after the jets acceptance 


\begin{tabular}{|c|c|c|c|c|}
\hline Cuts & Signal & $t \bar{t}+$ single top & $W b \bar{b} j j$ & $W W Z+W Z Z$ \\
\hline \hline Acceptance & 17.3 & $12.624 \times 10^{3}$ & 734.4 & 6.50 \\
\hline$H_{T}>500 \mathrm{GeV}, E_{T, m i s s}>60 \mathrm{GeV}$ & 13.7 & $2.807 \times 10^{3}$ & 196.2 & 2.15 \\
\hline$\left|m_{j j}-m_{W}\right|<20 \mathrm{GeV},\left|m_{j j b}-m_{t}\right|<20 \mathrm{GeV}$ & 13.4 & $2.612 \times 10^{3}$ & 5.91 & 1.42 \\
\hline$m_{b l}>180 \mathrm{GeV}$ & 9.26 & 79.1 & 3.92 & 0.12 \\
\hline$\Delta R_{t b}<1.6$ & 7.62 & 11.5 & 2.74 & $<10^{-2}$ \\
\hline$\left|m_{h a d}-m_{H^{ \pm}}\right|<20 \mathrm{GeV}$ & 7.00 & 2.07 & 0.38 & $<10^{-3}$ \\
\hline
\end{tabular}

Table I: The effect of the various levels of cuts devised to separate the signal from a $800 \mathrm{GeV}$ $Z^{\prime}$ decaying into a $300 \mathrm{GeV} H_{1}^{ \pm}$and a $W$ boson from the standard model backgrounds. In the third column is the QCD background, and in the fourth and fifth columns are the electroweak backgrounds. The $b$ tagging and lepton efficiencies were not taken into account yet. Gaussian smearing of energies and momenta (only the magnitude, not directions) are included in all rows.

cuts reaching several nanobarns. Assuming a bottom miss-tagging factor against light quark and gluons jets of $5 \times 10^{-3}$, the size of this background drops to the tens of femtobarns level. We checked that imposing the additional cuts virtually eliminates this source of backgrounds. Our simulations were performed at parton level though, and a more realistic computation including hadronization and showering and detector efficiencies will be necessary to confirm this claim; however we believe that the mass shell constraints are tight enough to clean the $W+4 j$ events.

Before looking for the charged Higgs resonance, a $S / B=0.54$ after applying all cuts can be achieved. A clear resonance in the $j j b \bar{b}$ invariant mass corresponding the production of a charged Higgs boson and subsequent decay to a top-bottom pair is visible over the total backgrounds for moderate Higgs masses from 200 to $300 \mathrm{GeV}$ at least as we show in the Fig. 18 below. The three signal lines in the $300 \mathrm{GeV}$ bin represent events from three different $Z^{\prime}$ masses: 800, 1000, and $1200 \mathrm{GeV}$. The peak from the $200 \mathrm{GeV}$ Higgs is the more pronounced but lies in a region richer in background events. The $400 \mathrm{GeV}$ resonance is the less pronounced mainly because of the $\Delta R_{t b}$ cut devised to eliminate the single top backgrounds. Despite the smaller phase space volume to produce heavy states, the $300 \mathrm{GeV}$ resonance is more favored than the $200 \mathrm{GeV}$ line even for very heavy $Z^{\prime}$ bosons once they yield harder jets and leptons which are more likely to pass the kinematical cuts. 


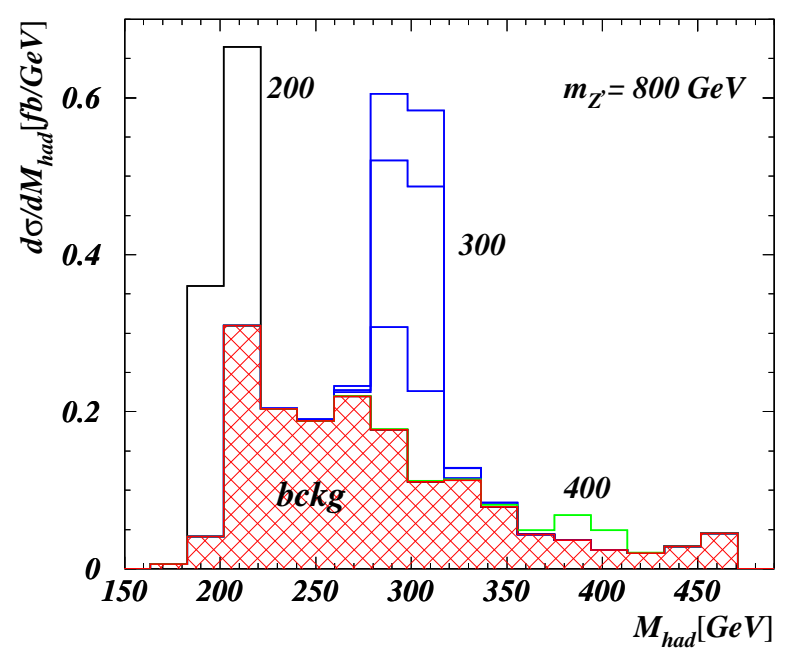

Figure 18: The invariant mass of the identified light quark and gluon jets plus the tagged bottom jets from the charged Higgs decay for the total backgrounds and the signal plus backgrounds. The empty histograms show the 200, 300, and $400 \mathrm{GeV}$ Higgs boson resonances for a $800 \mathrm{GeV} Z^{\prime}$ boson. For the $300 \mathrm{GeV}$ Higgs boson we also show the distributions for a 1 and $1.2 \mathrm{TeV}$ new neutral gauge boson represented by the lower lines.

Based on this distribution we compute the required luminosity for a statistically significant identification of the resonance associated to a charged Higgs boson production, looking for an excess of events in a window of $20 \mathrm{GeV}$ around the invariant mass of the identified jets which we call $M_{\text {had }}$. Recalling Eq. 31, $M_{\text {had }}$ is nothing but the invariant mass associated to the resonant structure $A$ from the charged Higgs boson decay.

Before the discussion of the LHC potential to search for these charged scalars we would like to emphasize that a more complete work including hadronization, showering, and extra radiation is needed to confirm our claims and estimate the impact of extra jets in the reconstruction of the Higgs resonance. Moreover, the NLO QCD effects including hard jet emission for signal and backgrounds would be an important improvement once the resonances are expected to show up in the tail of jets invariant mass distribution.

On the other hand some improvements can be devised in order to separate the signal and backgrounds even more efficiently, for example, if already exists a hint about the $Z^{\prime}$ mass scale. In this case, as we have seen, if the neutral gauge boson is heavy, is possible to impose much harder cuts on jets and leptons. In special, we checked that bottom transverse 
momentum is a good discriminant for very heavy states; however it must be kept in mind that hardening the bottom cuts could drastically decrease the $b$-tagging efficiency [30]. Moreover, it is easy to incorporate the signal semileptonic top quarks simply vetoing the hadronically decaying ones. In this case, the reconstructed charged scalars would suffer from a twofold ambiguity due the two momenta solutions for the neutrino momentum, but this is not an issue at all.

The Fig. 19 shows the integrated luminosity required for a $5 \sigma$ significance level observation based on the $S / \sqrt{S+B}$ statistics in the $m_{Z^{\prime}} \times m_{H_{1}^{ \pm}}$plane for $\lambda_{2}=0.31$ fixed based on the $M_{\text {had }}$ distribution shown at Fig. 18. The lower left corner of the masses plane $\left[600 \leq m_{Z^{\prime}} \leq 900\right] \times\left[200 \leq m_{H^{ \pm}} \leq 300\right]$ is the easier place for discovery as a consequence of the enhanced production cross section. A large portion of this corner demands only $15 \mathrm{fb}^{-1}$ of data at most for a $5 \sigma$ observation. The upper left corner $\left[600 \leq m_{Z^{\prime}} \leq 900\right] \times\left[300 \leq m_{H^{ \pm}} \leq 400\right]$, by its turn, is the less favored region for discovery mainly because of the $\Delta R_{t b}$ cut imposed to eliminate the single top backgrounds as we discussed earlier. A large portion of this corner cannot be probed even for $100 \mathrm{fb}^{-1}$ or more.

In the rest of this parameters space we observe the encouraging tendency to observe the heavier states. This is a direct consequence of the fact that heavy $Z^{\prime}$ bosons yield hard jets and leptons which are much more likely to pass cuts compared to the background events even considering the phase space suppression. It is worth noting again that the presence of a new gauge boson coupling to $W^{ \pm} H_{1}^{\mp}$ pairs, which is responsible for these singular kinematical configurations, is a distinguishing feature of the model as compared to MSSM and general 2HDM.

In the Fig. 20 right below we show the $S / B$ ratio as a function of the $Z^{\prime}$ mass for charged Higgses of 200, 300, and $400 \mathrm{GeV}$. The intermediate $300 \mathrm{GeV}$ Higgs bosons yield a $S / B>1$ for almost all $Z^{\prime}$ masses considered in this work. The 200 and $400 \mathrm{GeV}$ Higgses show a $S / B>1$ for light and heavy $Z^{\prime}$, respectively, and this is a consequence of our more or less blind set of cuts. As we discussed earlier the $\Delta R_{t b}$ cut favors large $m_{Z^{\prime}} \times m_{H_{1}^{ \pm}}$regions, but a more dedicated analysis can be made in order to observe the heavy Higgs and the light $Z^{\prime}$ portions of the parameters space. Note that the $400 \mathrm{GeV}$ case presents the larger $S / B$ ratios for heavy new gauge boson masses because of the much harder jets, bottoms, and leptons from their decays are much more likely to pass the cuts. On the other hand, the reduced production cross section demands more accumulated data for a significantly 


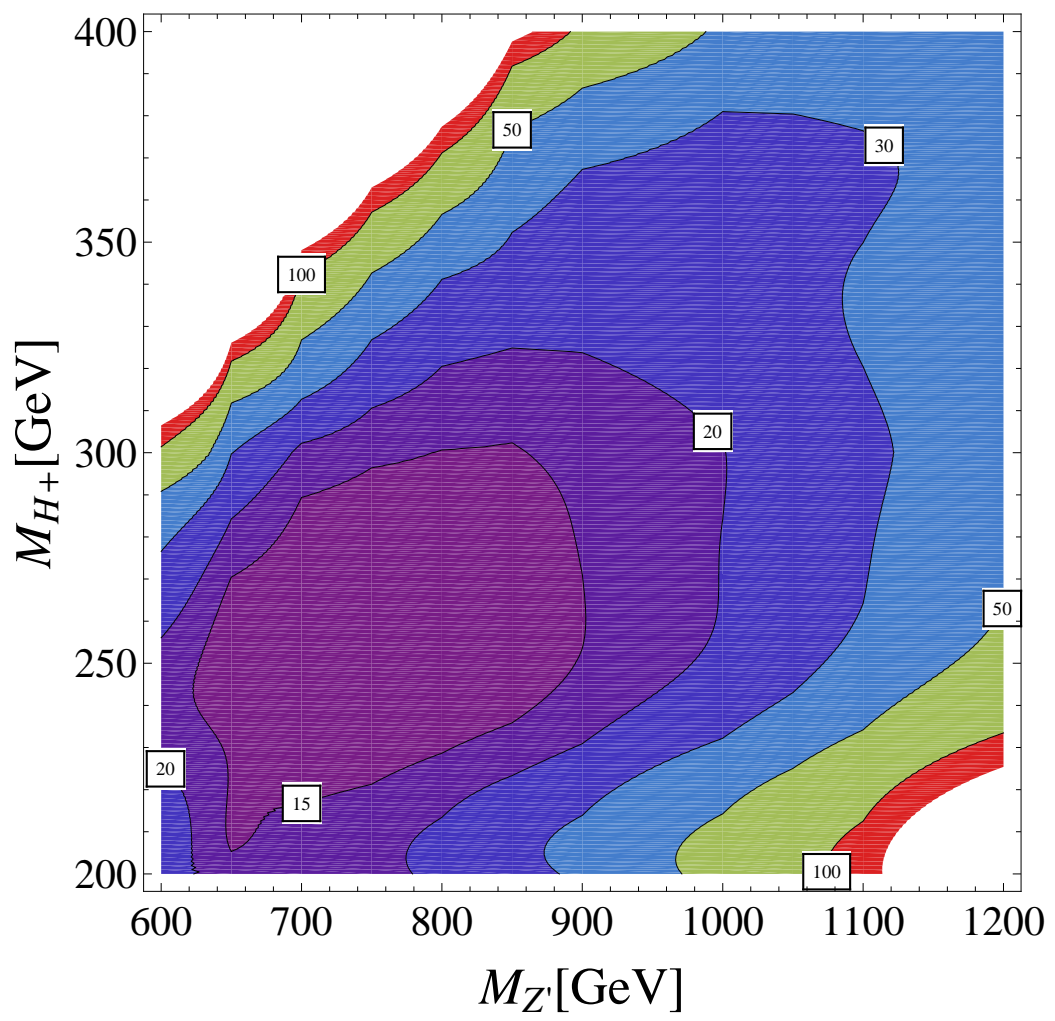

Figure 19: The required integrated luminosity for a $5 \sigma$ level statistical significant confirmation of the signal hypothesis over the background hypothesis in the $m_{Z^{\prime}} \times m_{H_{1}^{ \pm}}$plane. For this analyses we assumed $\lambda_{2}=0.31$.

statistical observations as can be seen in Fig. 19.

As a final remark we point out that $S / B \geq 1$ is a robust prediction in the sense it is less sensitive to systematic errors.

\section{CONCLUSIONS}

If the LHC collaborations could find experimental evidence for an scalar charged particle this will be an undisputed evidence of new physics beyond the SM. Moreover, it will shed light on the mechanism of electroweak symmetry breaking. In this work, we analyzed the scalar spectrum of the 3-3-1 model with right-handed neutrinos but with a custodial symmetry that reduces the number of parameters in the scalar potential. There are two charged and five neutral scalar states in the particle spectrum. The lightest charged Higgs bosons, $H_{1}^{ \pm}$, can be as light as $100 \mathrm{GeV}$. 


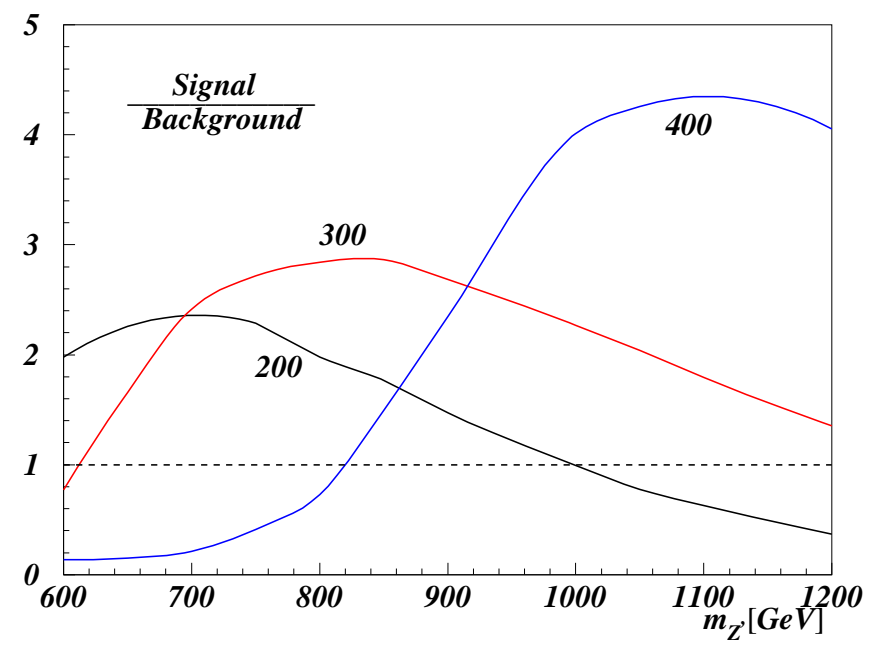

Figure 20: The $S / B$ ratio as a function of the $Z^{\prime}$ mass for three different charged Higgs masses: 200, 300, and $400 \mathrm{GeV}$.

The model presents several distinguishing features associated to the new gauge bosons and scalars. In special, there is an important interplay between one of the new neutral gauge bosons, $Z^{\prime}$, and the charged scalars of the model that enhances the production cross sections as compared to the MSSM or 2HDM. Yukawa interactions between the charged Higgses and heavy quarks are strong, which results in large cross sections for the typical production modes as the associated top-charged Higgs and charged Higgses from top quark decays. The charged Higgs pair production is also expected to be larger than its MSSM and 2HDM analogues as a consequence of the enhanced Yukawa interactions and the contribution from the new $Z^{\prime}$ gauge boson.

As an example of the role played by this new $S U(3)_{L}$ neutral gauge boson, the light charged Higgs bosons $H_{1}^{ \pm}$can be produced in association to a SM $W$ boson through the $Z^{\prime}$ decay. The charged Higgses decay into top and bottom quarks for $m_{H_{1}^{ \pm}}>m_{t}+m_{b}$, which lead to a $t b W \rightarrow b \bar{b} j j \ell \nu_{\ell}$ final state at hadron colliders as the LHC and the Tevatron. The top quark, the $W$ boson, and the charged Higgs resonances make the task to separate the signal from backgrounds relatively simple, and as a consequence a large portion of the parameters space of the model will be accessible to the $14 \mathrm{TeV}$ LHC Collaborations with up to $50 \mathrm{fb}^{-1}$, and even a $15 \mathrm{fb}^{-1}$ integrated luminosity would be just enough to discover a charged Higgs boson. We found that the same conclusion can not be claimed for the $7 \mathrm{TeV}$ 
LHC and the Tevatron because of the reduced amount of data designed for these experiments. Nevertheless the lighter charged Higgs, $m_{H_{1}^{ \pm}}<m_{t}+m_{b}$, decays predominantly to tau leptons and if the $Z^{\prime}$ is not too heavy this channel might be a good search channel for the Tevatron or even a $7 \mathrm{TeV}$ LHC in a longer run.

We did not try to optimize our analyses for very specific points of the parameters space, instead we focused on a more or less blind set of cuts taking into account only acceptance, trigger and tagging techniques. The robustness of the analyses shows up as signal to background ratio greater than 1 for a large portion of the parameters space under study in this

work. Detailed studies can be made though in order to take advantage of the hard jets and leptons expected for even heavier $Z^{\prime}$ and charged Higgses.

Acknowledgments: $\quad$ E. R. B. thanks PNPD-Capes and FAPESP for the financial support. A.G.D. also thanks FAPESP and CNPq for the financial support.

\section{Appendix A: Relevant Interaction Terms}

$$
\begin{gathered}
H_{1}^{+} W^{-} h_{1} \\
\mathcal{L}=-\frac{i \sqrt{2} g}{4} \frac{(u-v)}{v_{w}}\left[h_{1}^{0} \overleftrightarrow{\partial_{\mu}} H_{1}^{+}\right] W^{\mu-}+H . c \\
H_{1}^{+} W^{-} h_{2}=\frac{i \sqrt{2} g}{4} \frac{(u+v)}{v_{w}}\left[h_{2}^{0} \overleftrightarrow{\partial_{\mu}} H_{1}^{+}\right] W^{\mu-}+H . c \\
H_{1}^{+} H_{1}^{-} A_{\mu} \\
\mathcal{L}=-i e\left[H_{1}^{+} \overleftrightarrow{\partial_{\mu}} H_{1}^{-}\right] A^{\mu} \\
H_{1}^{+} H_{1}^{-} Z_{\mu} \\
\mathcal{L}=-\frac{i g}{2 \mathrm{cw}}\left(1-2 \mathrm{sw}^{2}\right)\left[H_{1}^{+} \overleftrightarrow{\partial_{\mu}} H_{1}^{-}\right] Z^{\mu} \\
H_{1}^{+} H_{1}^{-} Z_{\mu}^{\prime} \\
\mathcal{L}=-\frac{\mathcal{L}=\frac{g^{2} u v}{v_{w}} \frac{\mathrm{cw}^{3-\mathrm{sw}^{2}}}{\sqrt{3 g}} H_{1}^{+} W^{\mu-} Z_{\mu}^{\prime}}{\left(2\left(2 v^{2}+u^{2}\right) t^{2}-3\left(u^{2}-v^{2}\right)\right)\left[H_{1}^{+} \overleftrightarrow{\partial_{\mu}} H_{1}^{-}\right] Z^{\prime \mu}} \\
H_{1}^{+} W_{\mu}^{-} Z^{\prime \mu}
\end{gathered}
$$




$$
H_{2}^{+} H_{2}^{-} Z_{\mu}
$$

$$
\mathcal{L}=-\frac{i g}{2 \mathrm{cw}}\left[\frac{u^{2}}{\left(u^{2}+w^{2}\right)}-2 \mathrm{sw}^{2}\right]\left[H_{2}^{+} \overleftrightarrow{\partial_{\mu}} H_{2}^{-}\right] Z^{\mu}
$$

$H_{2}^{+} H_{2}^{-} Z_{\mu}^{\prime}$

$$
\mathcal{L}=\frac{i g}{2 \mathrm{cw}} \frac{\left(1-2 \mathrm{sw}^{2}\right)}{\sqrt{3-4 \mathrm{sw}^{2}}} \frac{u^{2}+2 w^{2}}{u^{2}+w^{2}}\left[H_{2}^{+} \overleftrightarrow{\partial_{\mu}} H_{2}^{-}\right] Z^{\prime \mu}
$$

[1] LEP Higgs Working Group for Higgs searches, hep-ex/ 0107031.

[2] CDF Collaboration, Phys. Rev. Lett. 96, 042003 (2006).

[3] K. A. Assamagan Y. Coadou and A. Deandrea, Eur. Phys. J. direct C 4, 9 (2002).

[4] CMS Collaboration, Report No. CERN/LHCC/94-38, (1994).

[5] F. Pisano and V. Pleitez, Phys. Rev. D 46, 410 (1992); P. H. Frampton, Phys. Rev. Lett. 69, 2889 (1992).

[6] J. C. Montero, F. Pisano, V. Pleitez, Phys. Rev. D 47, 2918 (1993); R. Foot, H. N. Long, T. A. Tran, Phys. Rev. D 50, R34 (1994); H. N. Long, Phys. Rev. D 53, 437 (1996).

[7] V. Pleitez, M. Tonasse, Phys. Rev. D 48, 2353 (1993).

[8] M. Singer, J. W. F. Valle, J. Schechter, Phys. Rev. D 22, (1980) 738.

[9] W. A. Ponce, Y. Giraldo, L. A. Sanches, Phys. Rev. D 67, (2003) 075001.

[10] C. A. de S. Pires, O. P. Ravinez, Phys. Rev. D 58, (1998) 35008; C. A. de S. Pires, Phys. Rev. D 60, (1999) 075013.

[11] E. Ramirez Barreto, Y. A. Coutinho and J. S Borges, Eur. Phys. J. C 50 ,909 (2007), ibid, Nucl. Phys. B 810, 210 (2009), ibid. Phys. Lett. B 689, 36 (2010); G. Tavares-Velasco, J. J. Toscano, Phys. Rev. D 65, (2002) 013005; L. Garcia-Luna, G. Tavares-Velasco, J. J. Toscano, Phys. Rev. D 69, (2004) 093005; M. S. Perez, G. Tavares-Velasco, J. J. Toscano, Phys. Rev. D 69, (2004) 115004; F. Ramires-Zavaleta, G. Tavares-Velasco, J. J. Toscano, Phys. Rev. D 75, (2007) 075008; J. G. Dueas, N. Gutierrez, R. Martínez, and F. Ochoa, Eur. Phys. J. C 60, $653(2009)$.

[12] A.E. Carcamo Hernandez, R. Martinez, F. Ochoa, Phys.Rev. D 73, 035007 (2006); J.M. Cabarcas, D. Gomez Dumm, R. Martinez, Eur. Phys. J. C 58, 569 (2008); J. E. Cieza Montalvo and M. Tonasse, Phys. Rev. D 71095015 (2005), J. E. Cieza Montalvo, N. V. Cortez, M. Tonasse, 
Nucl. Phys. B 756, 1 (2006), ibid. Phys. Rev. D 78, 116003, (2008) ; Le Duc Ninh and Hoang Ngoc Long, Phys.Rev. D 72, 075004 (2005); C. A. de S. Pires, P. S. Rodrigues da Silva, JCAP 0761, 012 (2007).

[13] A. G. Dias, J. C. Montero, V. Pleitez, Phys. Rev. D 73, 113004 (2006).

[14] R.M. Barnett, H.E. Haber and D.E. Soper, Nucl. Phys. B306, 697 (1988); A.C. Bawa, C.S. Kim and A.D. Martin, Z. Phys. C47, 75 (1990); V.D. Barger, R.J. Phillips and D.P. Roy, Phys. Lett. B324, 236 (1994); S. Moretti and K. Odagiri, Phys. Rev. D55, 5627 (1997); F. Borzumati, J.L. Kneur and N. Polonsky, Phys. Rev. D60, 115011 (1999); T. Plehn, Phys. Rev. D67, 014018 (2003); E.L. Berger, T. Han, J. Jiang and T. Plehn, Phys.Rev. D71, 115012 (2005); S.H. Zhu, Phys. Rev. D67, 075006 (2003); J. Alwall and J. Rathsman, JHEP 0412, 050 (2004); N. Kidonakis, JHEP 0505, 011 (2005).

[15] D.P. Roy, Czech.J.Phys. 55, B117 (2005), arXiv:hep-ph/0409201; V.M. Abazov et al. [D $\emptyset$ Collaboration], Phys. Rev. Lett. 88, 151803 (2002); S. Moretti and W.J. Stirling, Phys. Lett. B347, 291 (1995); A. Djouadi, J. Kalinowski and P.M. Zerwas, Z. Phys. C70, 435 (1996); E. Ma, D.P. Roy and J. Wudka, Phys. Rev. Lett. 80, 1162 (1998).

[16] A. Alves, T. Plehn, Phys. Rev. D 71, 115014 (2005).

[17] D. A. Dicus et al., Phys. Rev. D40, 787 (1989); A.A. Barrientos Bendez and B. A. Kniehl, Phys. Rev. D59, 015009 (1999); and Phys. Rev. D63, 015009 (2001); O. Brein, W. Hollik and S. Kanemura, Phys. Rev. D63, 095001 (2001); Z. Fei et al., Phys. Rev. D63, 015002 (2001); W. Hollik and S.H. Zhu, Phys. Rev. D65, 075015 (2002).

[18] J. Alwall et al., J. High Energy Phys. 09 (2007) 028.

[19] A.Pukhov and et al., CompHEP - a package for evaluation of Feynman diagrams and integration over multi-particle phase space. Users manual for version 3.3, hep-ph/9908288; E. Boos et al., [CompHEP Collaboration], CompHEP 4.4: Automatic computations from Lagrangians to events, Nucl. Instrum. Meth. A 534, 250 (2004). A. Pukhov, arXiv:hep-ph/0412191.

[20] J. Pumplin et al., JHEP 0602, 032 (2006).

[21] E. Boos and T. Plehn, Phys. Rev. D69, 094005 (2004).

[22] C.-X. Yue, Y.-Q. Di, Nucl. Phys. B 762, 55 (2006).

[23] S. Moretti, J. Rathsman, Eur. Phys. J. C 33, 41 (2004).

[24] A. Datta and A. Raychaudhuri, Phys. Rev. D62, 055002 (2000);

[25] S. M. Davidson and H.E. Logan, Phys. Rev. D82, 115031 (2010). 
[26] ATLAS Collaboration, ATLAS Technical Proposal, CERN/LHCC 94-43 (1994).

[27] CMS Collaboration, CMS Technical Design Report, CERN/LHCC 2006-21 (2006).

[28] T. Aaltonen et al. [CDF Collaboration], Phys. Rev. Lett. 106, 171801 (2011), arXiv: 1104.0699 [hep-exp].

[29] A. Alves, E. R. Barreto, A. G. Dias, work in progress.

[30] S. Gonzlez de la Hoz, L. March and E. Ros, ATL-PHYS-PUB-2006-003; L. March, E. Ros and B. Salvacha, ATL-PHYS-PUB-2006-002.

[31] Eri Asakawa, Oliver Brein and Shinya Kanemura, Phys. Rev. D 72, 055017, (2005). 